\title{
Cepstrum Coefficient Analysis from Low-Frequency to High-Frequency Applied to Automatic Epileptic Seizure Detection with Bio-Electrical Signals
}

\author{
Hao Ren ${ }^{1,2}{ }^{\mathbb{D}}$, Jianfeng $Q u^{1,2,3, *}$, Yi Chai ${ }^{1,2,3}$, Lei Huang ${ }^{4}$ and Qiu Tang ${ }^{1,2}$ \\ 1 Key Laboratory of Complex System Safety and Control, Ministry of Education, Chongqing University, \\ Chongqing 400044, China; renhao@cqu.edu.cn (H.R.); chaiyi@cqu.edu.cn (Y.C.); \\ tangqiu@cqu.edu.cn (Q.T.) \\ 2 School of Automation, Chongqing University, Chongqing 400044, China \\ 3 Collaborative Innovation Center for Brain Science, Chongqing University, Chongqing 400044, China \\ 4 School of Computer Science and Technology, Huaiyin Normal University, Huai'an 223300, China; \\ leihuang@hytc.edu.cn \\ * Correspondence: qujianfeng@cqu.edu.cn; Tel.: +86-23-6511-2173
}

Received: 2 July 2018 ; Accepted: 29 August 2018; Published: 1 September 2018

\begin{abstract}
This study analyzes bioelectrical signals to achieve automatic epileptic seizure detection. Electroencephalographic (EEG) signals were recorded with electrodes on healthy, epileptic seizure-free, and epileptic seizure patients. The challenges in this field are generally regarded to be the impacts of non-stationarity and nonlinearity in EEG signals. To address these challenges, this study attempts to recognize different brain statuses. The idea originated from a novel hypothesis that considers EEG signals as convolution signals and regards itself as the generation mechanism of EEG signals, to some extent. Based on this hypothesis, the nonlinear problem can be viewed as a deconvolution procedure. As such, the method can be simplified into three parts: eliminating non-stationary is used to catch high-frequency to low-frequency signals, which is followed by a local mean decomposition (LMD) algorithm; these signals are deconvoluted to form ultra-high-dimensional feature sets, which is completely terminated by the mel-frequency cepstrum coefficients (MFCC) algorithm; and several classifiers are combined to achieve highly accurate recognition results and to verify the superiority and reasonableness of this method. The publicly available EEG database from the University of Bonn, Germany is employed to demonstrate the effectiveness and outstanding performance of this method. According to the results, the method has the ability to attain a higher average classification accuracy than other methods in all of the four following cases: healthy (datasets $A$ and B) versus epileptic seizure (dataset E), epileptic seizure-free (datasets C and D) versus epileptic seizure (dataset E), healthy (datasets $A$ and $B$ ) versus epileptic seizure-free (datasets $C$ and D) versus epileptic seizure (dataset E), and healthy (dataset A) versus healthy (dataset B) versus epileptic seizure-free (dataset C) versus epileptic seizure-free (dataset D) versus epileptic seizure (dataset E).
\end{abstract}

Keywords: epileptic seizure detection; bioelectrical signals; local mean decomposition (LMD); mel-frequency cepstrum coefficients (MFCC)

\section{Introduction}

The demand has been increasing for implementing fast automatic epileptic seizure detection, shortening healing time, raising the success rate of cure, protecting patients from accidents, and saving their lives. As such, automatic epileptic seizure detection has become a hot research topic [1-5]. Recently, an incident report of epilepsy, one of the most common neurological disorders, stated that the prevalence is about 1 to $2 \%$ among the global population, and is rapidly increasing in the elderly 
population [1,4-6]. In the past several decades, although new anti-epileptic drugs had been invented, a third of epileptic patients are still suffering from epileptic seizures. Additionally, even though seizures are treated and controlled, self-reported life quality is significantly reduced by the unpredictability and consequences of epilepsy, as the disease causes many anomalies in terms of behavior, awareness, movement, and sensation $[3,7,8]$.

The significant differences between epileptic seizure and healthy states are generally highlighted as the frequency and patterns of neurons, meaning the spatial-temporal patterns of neurons gradually increase from the normal state to epileptic seizure-free state and then to the epileptic seizure state $[7,9]$. The World Health Organization (WHO) stipulates that epilepsy is caused by a group of brain cells with unexpected, uncontrolled electrical discharges, termed 'epileptic seizures' $[6,9,10]$. In 1929, Berger first measured the spontaneous electrical activity in the brain using electrodes, with electroencephalographic (EEG) signals being produced. His method is still considered a commonly used technology to monitor activity in the human brain. Afterwards, it was further used to detect epileptic seizure [9-14].

In the past and at present, well-trained and experienced neurophysiologists are employed universally to detect epileptic seizures through conventional methods by inspecting EEG signals over a long period $[6,9,10,13-16]$. Practically, to ensure high accuracy, detailed evaluations are essential, such as brain imaging tests like magnetic resonance imaging (MRI), computed tomography (CT), long-term EEG tests, blood tests, and medical history examination [15,17-24]. All of these evaluations are costly, which places a heavy financial burden on patients, especially those living in areas with frequent epileptic seizure breakout and poverty. Unfortunately, these methods have many limitations: for example, they are time-consuming, tedious, subjective, [9,10,13-16,25], and face many challenges in terms of inevitable factors on reducing misjudgments [13]. Computer-aided detection with EEG signals can therefore be considered a promising technology. It can be used to realize the goal of minimizing epileptic seizure activity to help control the disease $[16,26]$.

Recently, the signal-processing-based method has been applied to extract features to analyze, recognize, and detect epileptic seizures [1-5]. Between 2014 and 2017, many different methods were being used to extract and recognize features to achieve the automatic detection of epileptic seizures, such as transform techniques (e.g., discrete short-time Fourier transform, wavelet transform, generalized S-transform, shearlet and contourlet transforms), nonlinear dynamic analyses (e.g., various entropies and the Lyapunov exponent), and data-dependent time-frequency decomposition approaches (e.g., empirical mode decomposition and local mean decomposition) [5,7,15,17-24,26-32].

However, although numerous methods have been effectively used to achieve the automatic detection of epileptic seizures, there are still some problems affecting improved recognition accuracy. This fact illustrates the nonlinear and non-stationary problem in EEG signals, which causes many challenges in analyzing the signals with conventional methods $[30,31,33]$. Due to these reasons, in this study, a novel hypothesis is proposed and applied to the publicly available EEG database of the University of Bonn, Germany (UoB). The results demonstrate the reasonableness and accuracy of our proposed method for detecting epileptic seizures.

The remainder of this paper is structured as follows: Section 2 outlines the materials and problem statement, and also highlights the main contributions. In Section 3, the methodology is described in detail, including the novel hypothesis, the Mel-Frequency Cepstrum Coefficients (MFCC) for well-solved nonlinear problems, local mean decomposition (LMD) for well-solved non-stationary problems, and a performance evaluation. The reasonableness and availability of our novel method is illustrated by figures in Section 4, which also outlines the promising results and some comparisons between this method and other methods. Finally, Section 5 draws a conclusion and lists probable future research directions. 


\section{Materials and Problem Statement}

We used the publicly available EEG database of the UoB to verify the effectiveness and the correctness of our method. This database is the benchmark that is always used to develop epileptic seizure detection systems [1-16,25-31,33-43].

\subsection{EEG Data Materials}

This database contains five datasets (A, B, C, D, and E), each of which has 100 single-channel EEG segments of $23.6 \mathrm{~s}$ duration for 4097 samples with sampling rates of $173.610 \mathrm{~Hz}$. Five healthy volunteers were invited to record their EEG signals using gold-plated surface electrodes placed according to the principles of the 10-20 international electrode placement standard. Healthy EEG signals in dataset A and dataset B were then produced. Similarly, the EEG signals of five epileptic seizure-free patients were also recorded during epileptic seizure-free trials using electrodes, which were placed opposite to the epileptogenic zone (dataset $C$ ) and within the epileptogenic zone (dataset $D$ ). The epileptic seizure-free EEG signals in datasets $C$ and $D$ were also produced, accordingly. Dataset $E$ describes the epileptic seizure signals, which were collected by placing the electrodes in the epileptogenic zone, as shown in Table 1 [1-16,25-31,33-43].

Table 1. The definitions and descriptions for the electroencephalographic (EEG) signals from the University of Bonn, Germany.

\begin{tabular}{llllll}
\hline \multirow{2}{*}{$\begin{array}{l}\text { Individual } \\
\text { Information }\end{array}$} & \multicolumn{2}{c}{ Five Healthy Individuals } & \multicolumn{3}{c}{ Five Epilepsy Patients } \\
\cline { 2 - 6 } State & Dataset A & Dataset B & Dataset C & Dataset D & Dataset E \\
\hline Electrode type & $\begin{array}{l}\text { Awake with } \\
\text { eyes open } \\
\text { (Healthy) }\end{array}$ & $\begin{array}{l}\text { Awake with } \\
\text { eyes closed } \\
\text { (Healthy) }\end{array}$ & Seizure-free & Seizure-free & $\begin{array}{l}\text { Seizure } \\
\text { activity }\end{array}$ \\
\hline Electrode place & $\begin{array}{l}\text { Surface } \\
\text { international } \\
\text { system }\end{array}$ & $\begin{array}{l}\text { 10-20 } \\
\text { international } \\
\text { system }\end{array}$ & $\begin{array}{l}\text { Intracranial } \\
\text { opposite to } \\
\text { epileptogenic } \\
\text { zone }\end{array}$ & $\begin{array}{l}\text { Intracranial } \\
\text { epileptogenic } \\
\text { zone }\end{array}$ & $\begin{array}{l}\text { Intracranial } \\
\text { epileptogenic } \\
\text { zone }\end{array}$ \\
\hline No. of channels & 100 & 100 & 100 & 100 & 100 \\
\hline Epoch duration & 23.6 & 23.6 & 23.6 & 23.6 & 23.6 \\
\hline
\end{tabular}

The sample segment of each dataset is shown in Figure 1. The five healthy volunteers were awake and relaxed with their eyes open (dataset A) or closed (dataset B). Therefore, the difference between sample segments A and B was minimal in terms of subjective understanding. Datasets C, $\mathrm{D}$, and $\mathrm{E}$ were recorded to diagnose epileptic seizure using intracranial electrodes. There were some differences between the sample segments from datasets $C$ and $D$. The sample segment from dataset E was a typical epileptic seizure EEG segment, which was initially confirmed by well-trained and experienced neurophysiologists. From a subjective perspective, the sample segment from dataset $\mathrm{E}$ was quite different from those of datasets A, B, C, and D, as shown in Figure 1.

It should be noted that each EEG segment can be considered as the minimum signal-processing unit, which replaces its tail of $1.84 \mathrm{~s}$ (about 16 sample points) to rebuild itself. In this article, the number of EEG segments was sufficiently large (about 1592 segments in each dataset, and 7960 segments in total) to verify the effectiveness and correctness of this method. The results reflect the EEG signal segment level instead of single sample-point factors. 


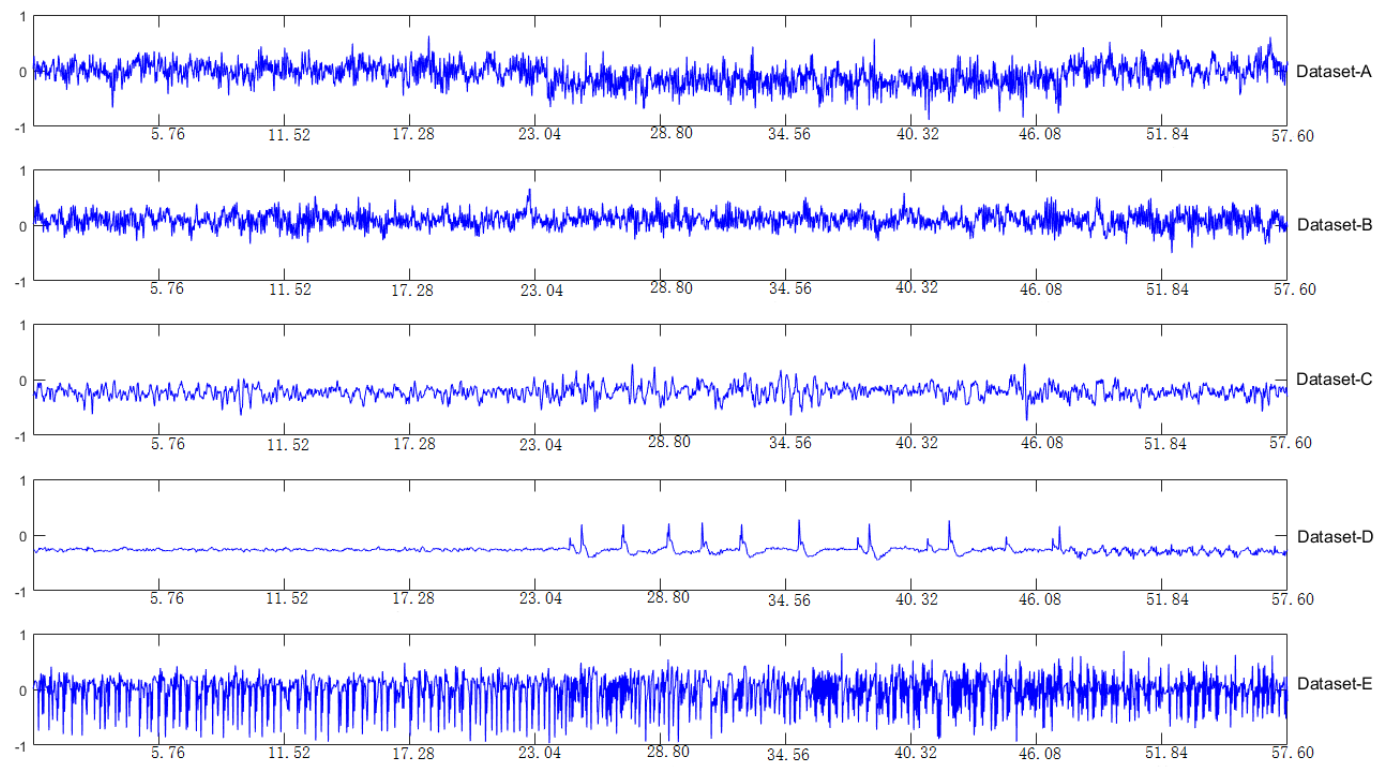

Figure 1. Typical electroencephalographic (EEG) segments from each of the five datasets (A-E).

\subsection{Problem Statement}

Four cases from the available database were considered. These cases were employed to investigate the difference between healthy, epileptic seizure-free, and epileptic seizure states in varying perspectives. These cases can be described as follows:

- Case I (Datasets A and, B versus dataset E): the recognition problem between healthy and epileptic seizure states. In this dataset, EEG segments from datasets A, B, and dataset E were employed, and they were divided into two categories: EEG segments belonging to datasets $\mathrm{A}$ and, $\mathrm{B}$, and EEG segments belonging to dataset $\mathrm{E}$.

- Case II (Datasets C and, D versus dataset E): the recognition problem between epileptic seizure-free and epileptic seizure states. In this dataset, EEG segments from datasets C, $\mathrm{D}$, and dataset $\mathrm{E}$ were employed, and they were divided into two categories: EEG segments belonging to datasets $C$ and, D, and EEG segments belonging to dataset $E$.

- Case III (Datasets A and B versus datasets C and D versus dataset E: used to determine the difference between the healthy, epileptic seizure-free, and epileptic seizure brain states. In this dataset, EEG segments from datasets A and B, EEG segments from datasets C and D, and EEG segments from dataset $\mathrm{E}$ were employed. These were divided into three categories: EEG segments belonging to datasets A and B, EEG segments belonging to datasets $C$ and D, and EEG segments belonging to dataset $\mathrm{E}$.

- $\quad$ Case IV (Dataset A versus dataset B versus dataset $C$ versus dataset $D$ versus dataset $E$ ): focused on an attempt to determine any slight changes in the EEG signals. In this dataset, EEG segments from datasets A, B, C, D, and E were employed, and were divided into five categories: EEG segments belonging to dataset $\mathrm{A}, \mathrm{EEG}$ segments belonging to dataset $\mathrm{B}, \mathrm{EEG}$ segments belonging to dataset C, EEG segments belonging to dataset $D$, and EEG segments belonging to dataset $\mathrm{E}$.

Almost all studies automatically classify EEG signals into either two classes: healthy (datasets A and B) and epileptic seizure-free (datasets C and D), or two classes: healthy (datasets A and B) and epileptic seizure (dataset E), or three classes: healthy (datasets A and B), epileptic seizure-free (datasets $C$ and D), and epileptic seizure (dataset E). Few studies have used five classes: healthy (two classes, datasets A and B), epileptic seizure-free (two classes, datasets C and D) and epileptic seizure (one class, dataset E). Simultaneously considering all of the above five situations is almost non-existent. 
Epilepsy, which involves sudden and often unforeseen occurrences, is a neurological condition in which sufferers experience spontaneous seizures that are caused by disturbances in the electrical activity of the brain $[1-5,44]$. In order to deal with these problems, a new theoretical perspective based on a hypothesis is proposed in this paper. The main contributions can be described as follows:

- The physical meanings of extracted features are very clear. To the best of our knowledge, this method is designed to explain the principle of EEG generation.

- The nonlinear and non-stationary problems are no longer an issue. The original intention of the hypothesis was to solve the nonlinear problem, which was proven by the later experiment results. Additionally, the important part, the LMD, was proven to be a good approach to deal with the non-stationary problem.

- Taking into account all of the recognition tasks. The above five situations were simultaneously considered to verify the correctness and effectiveness of this method, also reflect the generalization ability of EEG signal analysis.

\section{Methodology}

Generally, epileptic seizure detection can be formulated into a pattern recognition problem using the signal processing technique. Detection can be dismantled into three parts: feature extraction, selection, and feature recognition, as shown in Figure $2[1-5,44]$. The idea for this method originated from a novel hypothesis which considers EEG signals as convolution signals and regards itself as the generation mechanism of EEG signals. According to the two problems mentioned before, the LMD algorithm is often used to solve non-stationary problems, while MFCC is employed to solve nonlinear problems in EEG signals.

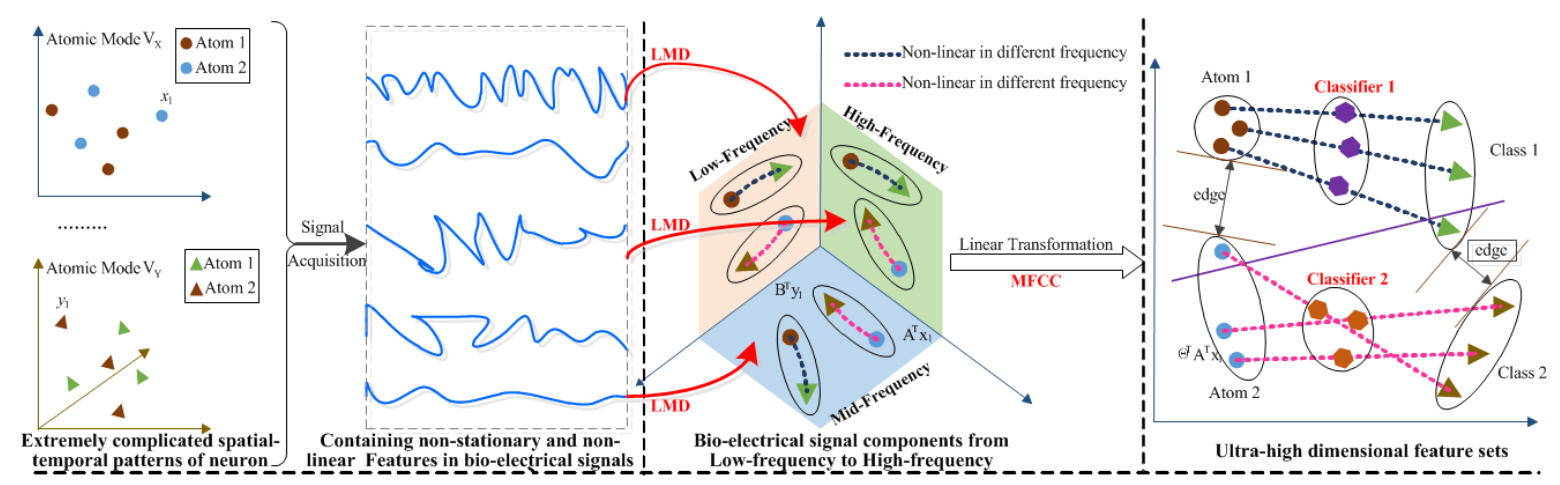

Figure 2. The architecture of the automated epileptic seizure detection approach.

The information provided in Figure 2 illustrates the architecture of the method used in this study. Bioelectrical signals were considered the interactions among a great number of "atomic modes", and the interactions were so complicated that they could not be described by existing mathematical language. Due to these extremely complicated spatial-temporal patterns of neurons, the bioelectrical signals often manifest with non-stationary and nonlinear characteristics, which were handled by LMD and MFCC in this article, respectively.

\subsection{Local Mean Decomposition (LMD) for Accurately-Solved Non-Stationary Problems in EEG Signals}

LMD is considered a data-dependent time-frequency decomposition approach with superior self-adaptive ability $[5,26,29,35,45]$. Our team previously completed a preliminary study on feature extraction based on LMD [5]. Following that study, LMD can be regarded as the most effective method based on time-frequency analysis from the highest frequency oscillation to the lowest frequency oscillation for non-stationary and nonlinear signals. Furthermore, the LMD method can be used to decompose EEG signals into a series of product functions (PFs). Then, the stationary statistical 
features of PFs can be obtained [26,45]. Figure 2 illustrates the first step of epileptic seizure detection, by decomposing the original EEG signals into several subcomponent signals to solve the non-stationary problem $[5,26]$.

Generally, EEG signals can be demodulated into PFs, and each PF can be considered as the product of the multiplication of an envelope signal by a frequency modulated (FM) signal. The LMD procedure involves iteratively extracting subcomponents from the highest to the lowest frequency oscillations, with low computational complexity and high processing speed $[5,26]$.

For an arbitrary signal $x(t)$, the iterative decomposition can be briefly described as follows [5,26]:

- $\quad$ Step 1 . All the local extreme points $n$ being determined, then the $i$-th local mean value $m_{i}$ of two adjacent extreme data points $\left(n_{i}\right.$ and $\left.n_{i+1}\right)$ can be calculated as follows.

$$
m_{i}=\frac{\left(n_{i}+n_{i+1}\right)}{2}
$$

From Equation (1), all the local means are straight lines extending between successive extremes. Then, an averaging operation can be carried out to smooth the block straight-lines. A smoothed local mean function $m_{11}(t)$ is therefore generated.

- Step 2. According to the above mentioned scenario, the local magnitude $a_{i}$ of two adjacent extremes $\left(n_{i}\right.$ and $\left.n_{i+1}\right)$ can be calculated as follows:

$$
a_{i}=\frac{\left|n_{i}-n_{i+1}\right|}{2} .
$$

Then, all local magnitudes can be plotted as straight lines extending as stated in Step 1, and an estimated envelope $a_{11}(t)$ can be derived.

- Step 3. The local mean function $m_{11}(t)$ is subtracted from the original signal $x(t)$, and can be described as follows, mathematically:

$$
h_{11}(t)=x(t)-m_{11}(t)
$$

$h_{11}(t)$ is then divided by the estimated envelope $a_{11}(t)$ and the demodulated component $s_{11}(t)$, which can be calculated as follows:

$$
s_{11}(t)=\frac{x(t)-m_{11}(t)}{a_{11}(t)}=\frac{h_{11}(t)}{a_{11}(t)} .
$$

- $\quad$ Step 4. Steps 1-3 are performed on the demodulated component for $n$ times until a purely FM element $s_{1 n}(t)$ is acquired. Under these circumstances, a series of recursive equations can be obtained as follows:

$$
\left\{\begin{array}{l}
h_{11}(t)=x(t)-m_{11}(t), \\
h_{12}(t)=s_{11}(t)-m_{12}(t), \\
\cdots \\
h_{1 n}(t)=s_{1(n-1)}(t)-m_{1 n}(t),
\end{array}\right.
$$

where

$$
\left\{\begin{array}{l}
s_{11}(t)=h_{11}(t) / a_{11}(t), \\
s_{12}(t)=h_{12}(t) / a_{12}(t), \\
\cdots \\
s_{1 n}(t)=h_{1 n}(t) / a_{1 n}(t) .
\end{array}\right.
$$

- $\quad$ Step 5. The estimated sub-envelopes $a_{11}(t), a_{12}(t), \ldots, a_{1 n}(t)$ are multiplied, and their integrated envelopes can be calculated as follows: 


$$
a_{1}(t)=a_{11}(t) a_{12}(t) \ldots a_{1 n}(t)=\prod_{q=1}^{n} a_{1 q}(t)
$$

where the ultimate objective can be described as follows:

$$
\lim _{n \rightarrow \infty} a_{1 n}(t)=1 .
$$

Generally, the first PF, named PF1, can be subsequently defined as the product of the multiplication of $s_{1 n}(t)$ by $a_{1}(t)$ :

$$
P F_{1}(t)=a_{1}(t) s_{1 n}(t) \text {. }
$$

- Step 6. PF1 is subtracted from the original signal $x(t)$, which turns into a new signal $u_{1}(t)$. Then, $u_{1}(t)$ can be considered as a new original signal and the whole procedure (Steps 1-5) can be repeated $k$ times until the $k$-th function $u_{k}(t)$ becomes a constant or contains no more oscillations:

$$
\left\{\begin{array}{l}
u_{1}(t)=x(t)-P F_{1}(t) \\
u_{2}(t)=u_{1}(t)-P F_{2}(t) \\
\cdots \\
u_{K}(t)=u_{k-1}(t)-P F_{k}(t) .
\end{array}\right.
$$

Finally, the original signal $x(t)$ can be decomposed into numerous PFs and a residual component $u_{k}(t)$. Furthermore, the original signal $x(t)$ can be reconstructed as follows:

$$
x(t)=\sum_{i=1}^{k} P F_{i}(t)+u_{k}(t)
$$

\subsection{Novel and Reasonableness Hypothesis for the Accurately-Solved Nonlinear Problem in EEG Signals}

In order to accurately solve the nonlinear problem in EEG signals, it is important to understand the work and generation mechanism of EEG signals. Currently, there are no definitive theories or mathematical models to describe the process of EEG production. However, some basic facts have been discovered, which are simplified and described in Figure 3. EEG signals are first produced by the nerve cells in the brain, as shown in Figure 3a. Usually, the characteristics of electrical signals are decided by two elements: the structure of brain cells, and motivation signals, as shown in Figure 3b. Neurons, connected by synapses, can be considered a type of cell that has a specific function of discharging electricity. The purpose of this electricity is to store and transmit information among different neurons. Neurons are often considered as the basic signal-processing unit of the human brain, whose main function is to receive information and transmit it to other cells, as shown in Figure 3a.

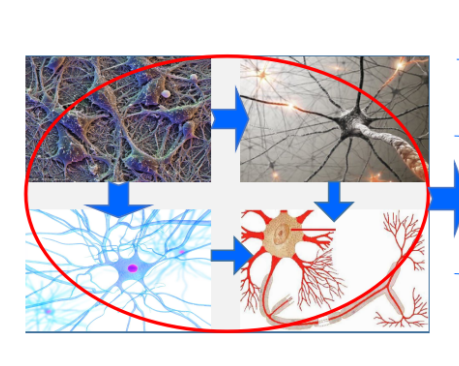

(a)

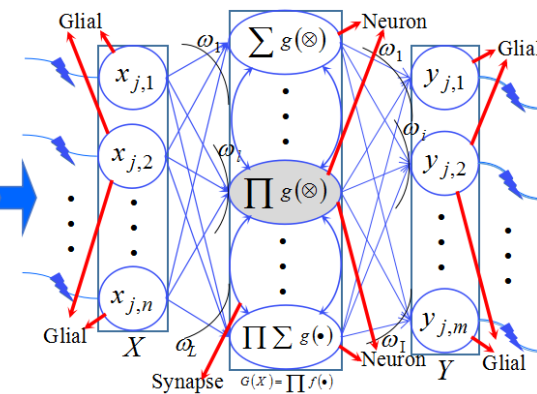

(b)

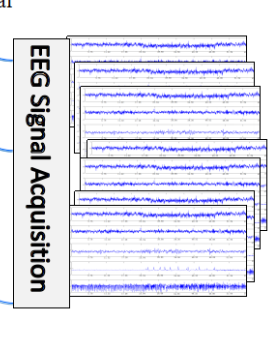

(c)

Figure 3. Hypothesis on the work mechanism of brain activity, (a) the nerve cells in the brain, (b) the structure of brain cells and motivation signals, and (c) collected electroencephalographic (EEG) signals. 
Figure $3 b$ demonstrates the hypothesis on the work and generation mechanism of EEG signals, which can be divided into three parts: motivation signals denoted by $x_{j, m}$, the working principle of neurons denoted by $g(\cdot)$, and the functional electrical signal output denoted by $y_{j, m}$. Clearly, the most important part is the working principle of neurons $g(\cdot)$, and, generally, each neuron is not isolated; they all connect with each other. Moreover, the connections are denoted by $\omega_{j, i}$, and the discharge function can be considered as two types of neurons: summation neuron $\sum$ and multiplication neuron $\prod$.

Usually, summation neuron $\sum$ refers to emphasizing signals according to linear functions, whereas multiplication neuron $\prod$ generates the new signals or enhances signal energy in accordance with some nonlinear pattern, which results in the EEG signals being nonlinear. Generally, the neuron design is a simple but extremely efficient architecture, which makes it extremely responsive to any minor electrical signals, and microscopically, it is non-stationary.

From the hypothesis on the construction of neurons, as shown in Figure 3b, functional electrical signal output can be expressed as follows:

$$
\begin{aligned}
y_{j, m} & =\prod g\left(w_{1} \cdot x_{1, n}\right) \oplus \prod g\left(w_{2} \cdot x_{2, n}\right) \oplus \ldots \oplus \prod g\left(w_{L} \cdot x_{j, n}\right) \\
& =\sum_{l=1}^{L}\left(\prod g\left(w_{l} \cdot x_{j, n}\right)\right),
\end{aligned}
$$

where $x_{j, n}, y_{j, m}$ denote the motivation signals and function electrical signal output, respectively, and $g(\cdot)$ is the working principle of neurons. It should be noted that the summation neuron $\sum$ was not considered because EEG signals are often non-stationary and nonlinear signals, which are generally caused by multiplication neurons $\Pi$. Therefore, it was reasonable and suitable for the following research work to analyze EEG signals for automated epileptic seizure detection. From the hypothesis, the following were assumed to be true: EEG signals are quite different in different individuals due to differences in the construction of brain cells and the characteristics of EEG signals recorded in different brain activities, which can be discriminated by the features denoted by the coefficient $\omega_{j, i}$.

The frequency spectra of EEG signals are always aliasing, and generally cannot be obtained by a series of simple filters. Deconvolution may be an efficient method to separate EEG signals into various components. One frequency segment, extracted from the original EEG signals, can be considered as the input signal of the brain neuron system, and can be calculated as follows:

$$
P F(n)=g_{1}(n) \times g_{2}(n),
$$

where $g_{1}, g_{2} \in R$ are the integration functions, which mean that the EEG signals from high frequency to low frequency can be regarded as the result of the convolution of two different functions, via the EEG signals being generated by the common effect of the neuronal structures and motivation signals. Therefore, the next step was to convert the convoluted signal into a time-domain linear signal.

We wish to illustrate is our hypothesis, which is the basis of this method. When solving different problems, LMD and MFCC were employed. All the methods used in this paper were built on this hypothesis instead of simply listing several methods, which is also the innovative contribution of this work. Accordingly, we refer to "this method" instead of "this proposed method" to avoid misunderstanding. To the best of our knowledge, this hypothesis can be regarded as a theoretical perspective that merits study.

MFCC was originally designed to study real speech registered by human ears. Since the frequency of speech signals is not linear, it requires the study of a new frequency unit that effectively represents the relationship between the size and the frequency. It must also satisfy the following three conditions: the general physical linear description, a low level of discrimination in the high frequency range, and a high level of discrimination in the low frequency range [41,43,46-51]. 
- Step 1. The PFs are segmented into $N$ frames. The EEG signals can be set with a $23.6 \mathrm{~s}$ duration to guarantee short-time stationarity inside a frame. Generally, there is an overlap with a duration of one-half of a frame between two adjacent frames [46-48,50,51]. The hamming window was applied to each frame to obtain windowed frames, and can be calculated as follows:

$$
H(k)=a-b \cos \frac{2 \pi k}{N-1}, k=0,1,2, \ldots, N-1,
$$

where $N$ is the number of points in a frame, and $a, b$ denotes the parameters of the hamming window $(a=0.54, b=0.46)$.

- $\quad$ Step 2. The frequency spectrum $F(\omega)$ of the $i$-th frame $x_{i}(n)$ can be calculated using the Fast Fourier Transform. The short-time power spectrum $|F(\omega)|^{2}$ can be calculated and filtered by a Mel-filter bank $B_{M e l}$, which consists of $M$ triangular band-pass filters and is located in the Mel-frequency domain in a uniformly spaced manner. The mapping relation from a linear frequency $f$ to Mel-frequency $f_{M e l}$ can be calculated by the following equation:

$$
f_{\text {Mel }}=\delta \ln (1+f / v)
$$

where $f, f_{\mathrm{Mel}}$ denote the linear frequency and Mel-frequency, respectively, and $\delta, v$ are the parameters $(\delta=2595$, and $v=700)$.

- Step 3. Then, the output of the short-time power spectrum $|F(\omega)|^{2}$ can be calculated via this Mel-filter bank:

$$
\theta\left(M_{k}\right)=\ln \left[\sum_{k=1}^{N}|X(k)|^{2} H_{m}(k)\right], m=1,2, \ldots, M .
$$

- Step 4. Finally, the output power $\theta\left(M_{k}\right)$ of $m$-th can be calculated, and the Mel-Frequency Cepstrum $c(k)$ can be calculated by applying the updated Inverse Discrete Cosine Transform (IDCT) in the Mel-frequency coordinate spectrum, which can be described as follows, mathematically:

$$
c_{k}=\sum_{k=1}^{M} \theta M_{k} \cos \left(\frac{n(l-0.5) \pi}{M}\right), n=1,2, \ldots, p,
$$

where $p$ is the dimension of MFCC, $c(k)$ denotes the $k$-th MFCC, and $p$ is less than the number $M$ of Mel-filters.

\subsection{Identification Mechanism-Categorical Classifications}

Practical experience has demonstrated that the performance of epileptic seizure detection depends not only on feature extraction but also on the quality of features selected from EEG data. This procedure is often hidden in the categorical classification. The correlation among features is a good break point to achieve the reduction of feature dimension $[8,15,16,32,41,43,52-54]$. Recognition, also called classification, can be considered an important research field, whose common and traditional methods mainly include support vector machine (SVM), K-nearest neighbor (KNN), neural network (NN) classifiers, forest classifiers, and decision trees (DTs) [8-10,14,25-60].

In the present work, a series of coefficient features, calculated using the MFCC algorithm, were gathered for classification using a wide range of classifiers. The recognition of EEG signals was perfectly achieved. In order to test and verify the effectiveness and correctness of our proposed hypothesis, multiple classification categories were employed in the current study, such as back-propagation neural network (BPNN), SVM, and KNN.

- Back-Propagation Neural Network (BPNN) is a feed-forward neural network that can be considered a category of artificial neural networks (ANNs). Generally, the structure of BPNN contains three layers: an input layer, a hidden layer, and an output layer. The steepest descent 
algorithm is often employed in the learning rule, and the weights and thresholds can be gradually adjusted, so the minimum summation of squared errors can be reached [5,26,32].

- Support Vector Machine (SVM) is a potential methodology used for solving the classification problem. It is not only regarded as a linear classifier, but also as a nonlinear classifier. Usually, SVM can be employed to minimize the operational error and maximize the margin hyperplane to maximize the classification performance $[5,7,9,10,14,25,27,35]$.

- Linear Discriminant Analysis (LDA) has been successfully used in multidimensional reduction and pattern recognition [26,41]. LDA can be defined as classifying the dependence by separating the $m$-dimensional descriptor space into two areas that are separated by a hyperplane. Concerning classification issues, LDA can be used to minimize the distances among vectors of the same class and maximize the distance of inconsistent class centers [26,41].

- K-nearest neighbour (KNN) is often used to identify different EEG signals, and can be considered as a supervised and non-parametric machine learning technique, which is relatively simple, has low computational burden, and is easy to implement $[5,11,26,40]$.

- Decision Tree (DT) has been verified to have a high interpretability, and the rules, obtained by splitting the training dataset into subsets by testing attributes, are also readily compressible [39].

In this paper, the recognition accuracy is calculated and simplified first as a summation and then as the average value, which can be described as follows:

$$
\text { Aver. }=\frac{1}{N} \sum_{i=1}^{N} y_{i}
$$

where $y_{i}$ represents the classification result of the $i$-th classifier, and $N$ denotes the number of classifiers, and Aver. denotes the recognition accuracy.

The goal of simultaneously using different types of classifiers involves two points: to attempt to mix several classifiers to obtain a stable result, and to verify the super generalization ability of EEG signal analysis. Once all recognition results were calculated for each testing dataset, and the average value for all was calculated, the result was more credible with universal significance.

\subsection{Performance Evaluation-Confusion Matrix Metrics}

Confusion matrix metrics are often used to quantitatively evaluate recognition performance. A confusion matrix generally involves the following parameters:

- $\quad \mathbf{a}$ is the number of healthy EEG records identified correctly,

- $\quad \mathbf{b}$ is the number of healthy EEG records mistakenly detected as epilepsy,

- $\quad \mathrm{c}$ is the number of epileptic EEG data mistakenly identified as healthy,

- $\mathbf{d}$ is the number of epileptic EEG data detected correctly.

The following aggregate metrics can then be calculated based on the above parameters.

- Accuracy (AC), the proportion of total number of correct predictions, is $(a+d) /(a+b+c+d)$.

- True positive rate (TP), the proportions of positive cases correctly identified, is $d /(c+d)$.

- False positive rate (FP), the proportion of negatives cases classified incorrectly, is $a /(a+b)$.

- True negative rate (TN), the proportion of negatives cases classified correctly, is $a /(a+b)$.

- False negative rate (FN), the proportion of positives cases classified incorrectly, is $c /(c+d)$.

- Precision (P), the proportion of the correctly predicted positive cases, is $d /(b+d)$.

- Sensitivity (SEN) is $T P /(T P+F N) \times 100 \%$, specificity(SPE) is $T N /(T N+F P) \times 100 \%$.

The criteria for performance evaluation are usually employed in biomedical studies, which include three parts: sensitivity (the proportion of the total number of labeled ictal EEGs that are correctly classified), specificity (the proportion of the total number of labeled inter-ictal EEGs that are correctly 
classified), and classification accuracy (the proportion of the total number of EEG signals that are correctly classified) $[2,6-10,13-16,25-32,34,35,38-41,49,53,59]$.

\section{Results and Discussion}

This section describes the results of the publicly available UoB EEG database to show the effectiveness and correctness of the method.

\subsection{Experimental Setup}

The majority of experiments used the train-to-test ratio (TTTR) of 7:3 in each EEG channel, meaning that $70 \%$ of the dataset was used for training, and the remaining $30 \%$ was used for testing. Many researchers have achieved the estimated performance using a 9:1 ratio, which makes the performance predictably high with such a high percentage of datasets for training. To the best of our knowledge, varying the TTTR merits investigation to test the robustness of the computational performance [6]. Therefore, this experiment set the TTTRs as 9:1, 8:2, 7:3, 6:4, 5:5, 4:6, 3:7, 2:8, and 1:9.

This study implemented the algorithm using the MATLAB (R2017a) software (MathWorks, Natick, Massachusetts, USA) and its toolboxes, on a computer with a $3.20 \mathrm{GHz}$ Intel-Core-i5-3470 CPU (Beijing, China), with 12 GB of RAM and running 64-bit Windows7.

In order to eliminate the influence of different time-space dimensions, the data had to be processed by normalization methods. After that, the features were of the same order of magnitude and the data were then suitable for comprehensive comparison and evaluation. This experiment was carried out on EEG signals using the following equation, which is usually called the Z-core normalization method:

$$
x_{i}^{*}(t)=\frac{x_{i}(t)-\mu}{\sigma}
$$

where $\mu, \sigma$ are the mean and standard deviation of sample EEG segment $x_{i}(t)$, respectively, and $x_{i}^{*}(t)$ is the normalized $x_{i}(t)$.

\subsection{Signal Subcomponents (Product Functions (PFs)) Solving the Non-Stationary Problem}

The iterative decomposition algorithm LMD has shown great potential for handling various kinds of signals. However, the number of obtained PFs is not completely consistent when applied to EEG signals. This phenomenon may be caused by the endpoint effect during the decomposition procedure. Therefore, an approach known as the mirror extending technique was applied to address this problem. This technique involves the boundaries of signals at both ends being counted as two symmetrical axes, so that the original segment can be stretched into a triple-length sequence. Finally, the LMD can be subsequently executed on this extended sequence.

Sample EEG signals of these five EEG datasets (datasets A, B, C, D, and E) and their corresponding PFs (PF1 to PF5) are shown in Figure 4. The subcomponents from top to down are denoted by PF1 to PF5, and PF1 generally contains more high-frequency elements than the others. Considerably more noise is always contained in the highest frequency component, and similarly the lowest component always contains large amounts of useless information. The decomposed PFs of each EEG segment were considered as the high frequency component (PF1) to the low frequency component (PF5). Therefore, the subcomponents from PF1 to PF5, meaning from high-frequency to low-frequency components, were employed to represent the functional information generated by one or several neurons. 
Set-A
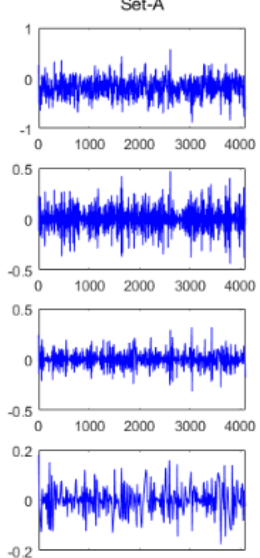

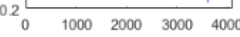

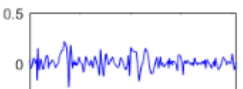

\begin{tabular}{lllll}
\hline 0.5 & & & & \\
0 & $1000 \quad 2000$ & 3000 & 4000
\end{tabular} 0.2

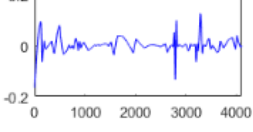

Set-B
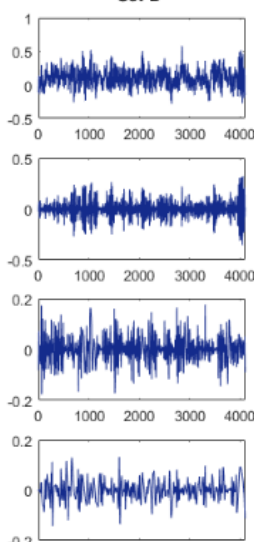

$\begin{array}{lllll}-0.2 & 1000 & 2000 & 3000 \quad 4000\end{array}$
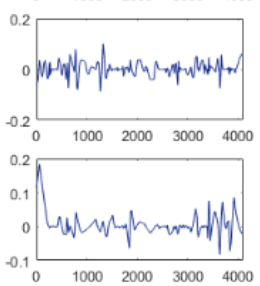

Set-C
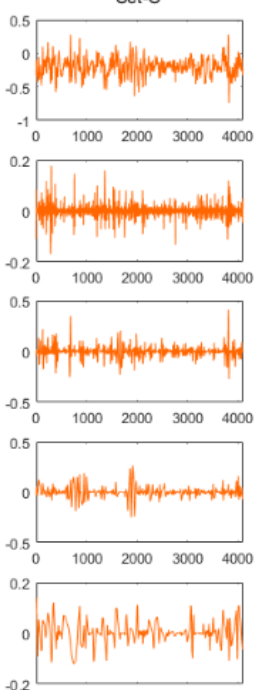

$\begin{array}{rrrrr}-0.2 & & & & \\ 0 & 1000 & 2000 & 3000 & 4000\end{array}$

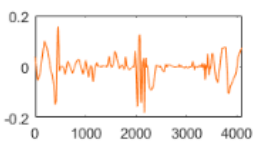

Set-D
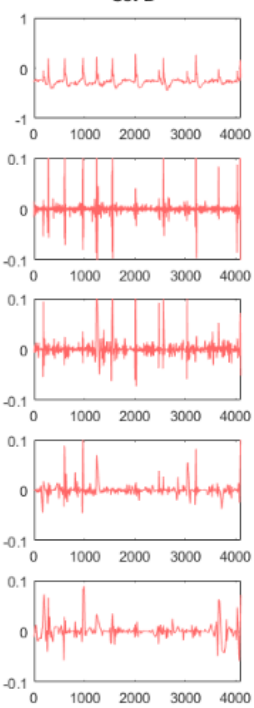

$\begin{array}{lllll}0.1 & 1000 & 2000 & 3000 & 4000\end{array}$

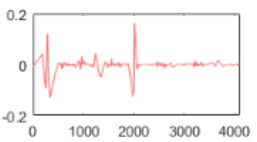

Set-E

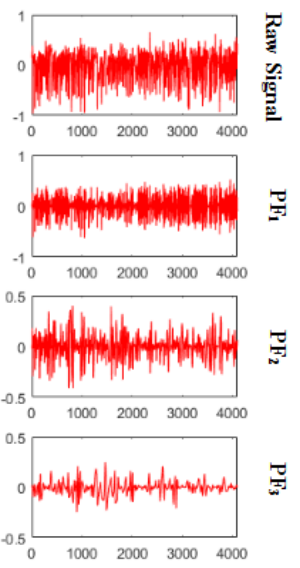

$\exists$

赵

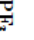

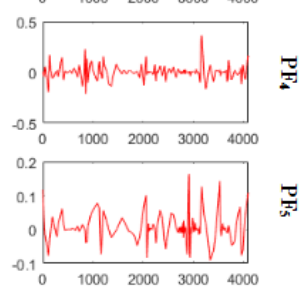

Figure 4. Sample Electroencephalographic (EEG) segments of five datasets (datasets A-E) and their corresponding product functions ( $\mathrm{PFs}$ ) (PF1 to PF5).

\subsection{Mel-Frequency Cepstrum Coefficients (MFCC) Solving the Nonlinear Problem}

The EEG signals can be deconvoluted into a time-domain signal using the MFCC algorithm; its first series of typical coefficient results are provided in Figures 5 and 6. The MFCCs in PF1 from top to down are clearly very different. It is foreseeable that the MFCCs can be fed into some classifiers, and then the classifiers, containing hundreds of dimensional features, will be built up and can be used to achieve a super recognition result.

Generally, as mentioned before, the first subcomponent PF1 contains more information than the others, and this opinion was verified by the results of five datasets (datasets $A, B, C, D$, and E), as shown in Figure 5, and the differences among these five datasets (datasets A, B, C, D, and E) are obvious in MFCC (PF1). The MFCCs of the extracted features are not just these exhibitions, but were numerous (about 144 features from PF1 to PF4). Furthermore, when the datasets were fed into some classifiers, the recognition results (provided in the next subsection in details) were obtained quickly with impressive results. To explaining the causes of this phenomenon, we need to return to our hypothesis. The construction of brain cells is often unique and the motivation signals are large in number. This is consistent with the result obtained from the current biomedical research.

Although there are some cluttered features that cannot be distinguished, the results of these five different types of classifiers demonstrate the efficiency of these features. One possible explanation is that the dimensions of these 144 features can be employed to form ultra-dimensional spatial-temporal space, which is very hard to describe. Figures 5 and 6 best describe what we know so far. 

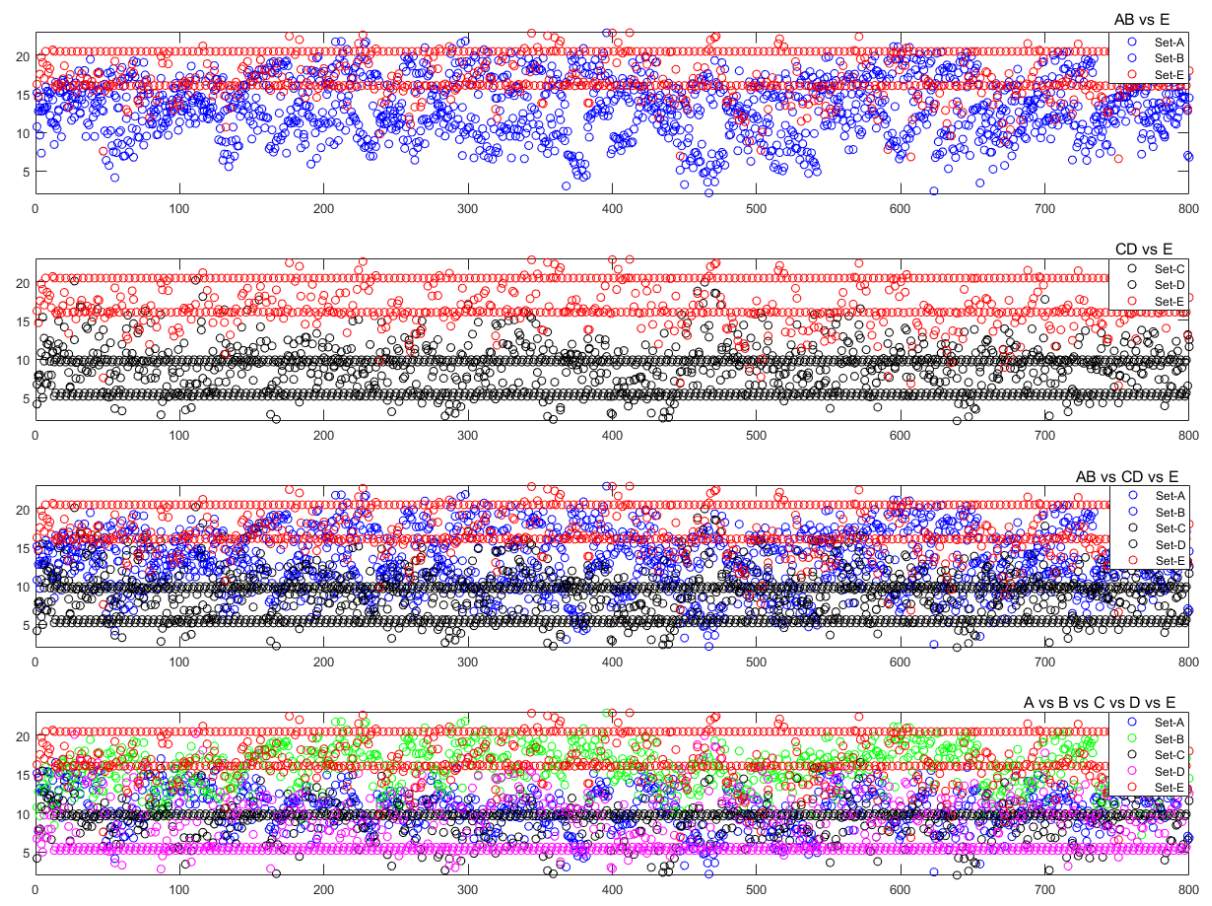

Figure 5. The first series of Mel-frequency cepstrum coefficients (MFCCs) in product function1 (PF1) of five sets (sets A, B, C, D, and E), for Case 1 to Case 4 from top-down.
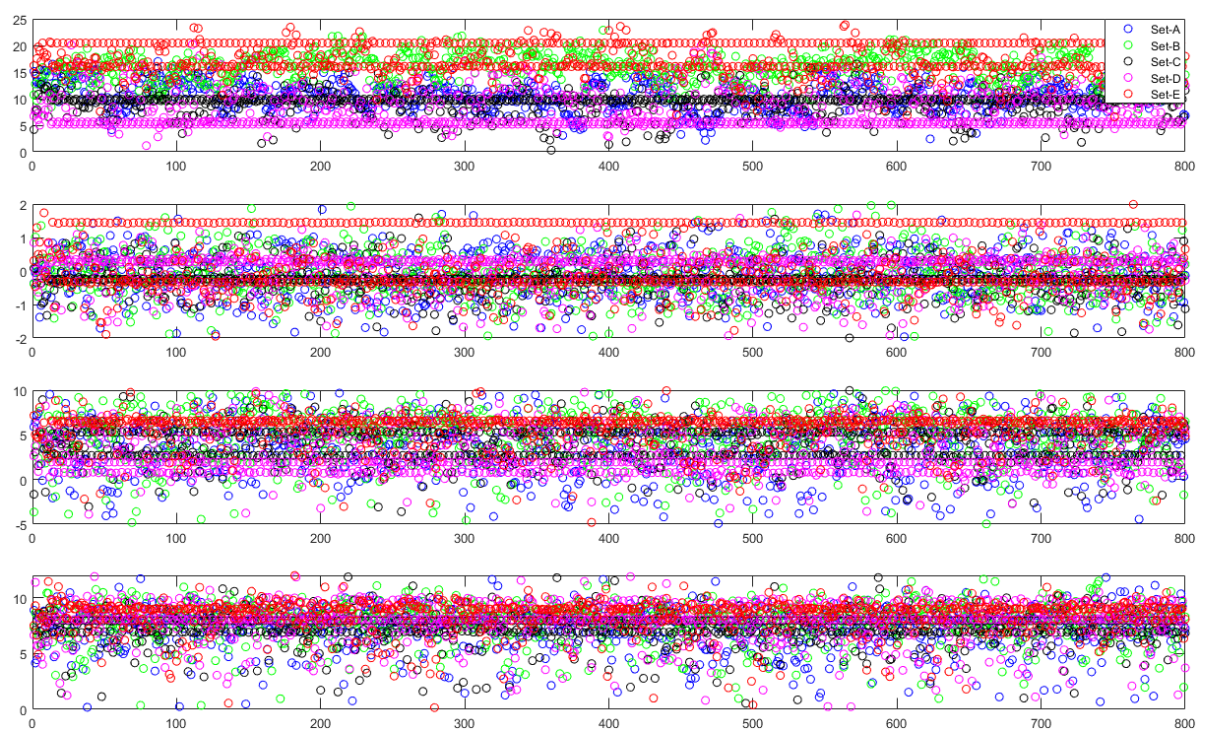

Figure 6. The first series of Mel-frequency cepstrum coefficients (MFCCs) of five sets (sets A, B, C, D, and $\mathrm{E}$ ) in Case 4, for product function1 (PF1) to product function4 (PF4) from top-down.

\subsection{Recognition Result}

In this study, a total of 6368 EEG segments were considered, and can be considered as the most difficult recognition problem. Systematic analysis seems highly necessary, and accordingly the experiment was conducted by considering all the classification problems, using the four cases, which completely contained all the possible situations.

In order to evaluate the performance of this method with the four cases, Table 2 illustrates the classification results with the five classifiers. Based on the results, the highest in Case 1 was 100\%, which means that the combination, created with the characteristics as the key features in all the experiments, can be employed to obtain a perfect recognition result. 
The results in Table 2 suggest that Case 1 (healthy versus epileptic seizure) yielded a high accuracy by this method. In order to assess the performance of this proposed method, other cases were also used and classified, as shown in Tables 3-6. The results can be used to provide enough evidence to show the efficiency of this method on extracting the useful features.

The results in Table 3 demonstrate the detection ability for healthy EEG groups versus epileptic seizure groups, whereas Table 4 illustrates the results of the epileptic seizure-free versus epileptic seizure groups. Table 5 describes the three classification problem for healthy, epileptic seizure-free, and epileptic seizure EEG segments. Table 7 tries to explain all the differences between the five EEG datasets. It should be noted that all of the classification problems were solved perfectly.

Table 2. Classification results of four cases on all data-sets (Back-Propagation Neural Network (BPNN), Support Vector Machine (SVM), Linear Discriminant Analysis (LDA), K-nearest neighbour (KNN), Decision Tree (DT), average value (Aver.)).

\begin{tabular}{ccccccc}
\hline Cases & BPNN & SVM & LDA & KNN & DT & Aver. \\
\hline I & $\mathbf{1 0 0}$ & $\mathbf{1 0 0}$ & $\mathbf{1 0 0}$ & 99.89 & 99.91 & $\mathbf{9 9 . 9 6}$ \\
II & $\mathbf{1 0 0}$ & $\mathbf{1 0 0}$ & 99.93 & $\mathbf{1 0 0 . 0}$ & 97.56 & $\mathbf{9 9 . 5 0}$ \\
III & $\mathbf{1 0 0}$ & 99.71 & 98.83 & 97.80 & 94.66 & $\mathbf{9 8 . 1 8}$ \\
IV & $\mathbf{9 8 . 3 5}$ & 95.27 & 93.05 & 88.94 & 90.95 & $\mathbf{9 3 . 3 1}$ \\
\hline
\end{tabular}

Table 3. Classification results of the five classifiers on all five data-sets of Case 1 (Back-Propagation Neural Network (BPNN), Support Vector Machine (SVM), Linear Discriminant Analysis (LDA), K-nearest neighbour (KNN), Decision Tree (DT), average value (Aver.)).

\begin{tabular}{cccccccc}
\hline Sets & Class & BPNN & SVM & LDA & KNN & DT & Aver. \\
\hline \multirow{2}{*}{ A } & Healthy & $\mathbf{9 9 . 9 7}$ & $\mathbf{1 0 0 . 0}$ & $\mathbf{1 0 0 . 0}$ & $\mathbf{9 9 . 9 4}$ & $\mathbf{1 0 0 . 0}$ & $\mathbf{9 9 . 9 8}$ \\
& Epileptic & 0.03 & 0.00 & 0.00 & 0.06 & 0.00 & 0.045 \\
\hline \multirow{2}{*}{ B } & Healthy & $\mathbf{9 9 . 9 7}$ & $\mathbf{1 0 0 . 0}$ & $\mathbf{1 0 0 . 0}$ & $\mathbf{9 9 . 7 5}$ & $\mathbf{9 9 . 9 4}$ & $\mathbf{9 9 . 9 3}$ \\
& Epileptic & 0.03 & 0.00 & 0.00 & 0.25 & 0.06 & 0.170 \\
\hline \multirow{2}{*}{ C } & Healthy & $\mathbf{9 8 . 8 7}$ & $\mathbf{9 9 . 3 1}$ & $\mathbf{9 9 . 6 2}$ & $\mathbf{9 8 . 6 8}$ & $\mathbf{9 8 . 8 1}$ & $\mathbf{9 9 . 0 6}$ \\
& Epileptic & 1.17 & 0.69 & 0.38 & 1.32 & 1.19 & 0.950 \\
\hline \multirow{2}{*}{ D } & Healthy & $\mathbf{9 8 . 9 0}$ & $\mathbf{9 9 . 6 9}$ & $\mathbf{9 9 . 7 5}$ & $\mathbf{9 7 . 7 4}$ & $\mathbf{9 7 . 8 0}$ & $\mathbf{9 8 . 7 8}$ \\
& Epileptic & 1.10 & 0.31 & 0.25 & 2.26 & 2.20 & 1.224 \\
\hline \multirow{2}{*}{ E } & Healthy & 0.19 & 0.00 & 0.06 & 0.00 & 0.44 & 0.138 \\
& Epileptic & $\mathbf{9 9 . 8 1}$ & $\mathbf{1 0 0 . 0}$ & $\mathbf{9 9 . 9 4}$ & $\mathbf{1 0 0 . 0}$ & $\mathbf{9 9 . 5 6}$ & $\mathbf{9 9 . 8 6}$ \\
\hline
\end{tabular}

Table 4. Classification results of the five classifiers on all five data-sets of Case 2 (Back-Propagation Neural Network (BPNN), Support Vector Machine (SVM), Linear Discriminant Analysis (LDA), K-nearest neighbour (KNN), Decision Tree (DT), average value (Aver.)).

\begin{tabular}{cccccccc}
\hline Sets & Class & BPNN & SVM & LDA & KNN & DT & Aver. \\
\hline \multirow{2}{*}{ A } & Inter-ictal & $\mathbf{9 8 . 4 6}$ & $\mathbf{9 9 . 3 1}$ & $\mathbf{9 8 . 7 4}$ & $\mathbf{9 0 . 7 0}$ & $\mathbf{9 4 . 4 1}$ & $\mathbf{9 6 . 3 2}$ \\
& Epileptic & 1.54 & 0.69 & 1.26 & 9.30 & 5.59 & 3.676 \\
\hline \multirow{2}{*}{ B } & Inter-ictal & $\mathbf{9 0 . 2 3}$ & $\mathbf{9 3 . 5 3}$ & $\mathbf{8 8 . 6 3}$ & $\mathbf{7 7 . 9 5}$ & $\mathbf{7 6 . 7 6}$ & $\mathbf{8 5 . 4 2}$ \\
& Epileptic & 9.77 & 6.47 & 11.37 & 22.05 & 23.24 & 14.58 \\
\hline \multirow{2}{*}{ C } & Inter-ictal & $\mathbf{1 0 0 . 0}$ & $\mathbf{1 0 0 . 0}$ & $\mathbf{1 0 0 . 0}$ & $\mathbf{1 0 0 . 0}$ & $\mathbf{1 0 0 . 0}$ & $\mathbf{1 0 0 . 0}$ \\
& Epileptic & 0.00 & 0.00 & 0.00 & 0.00 & 0.00 & 0.00 \\
\hline \multirow{2}{*}{ D } & Inter-ictal & $\mathbf{1 0 0 . 0}$ & $\mathbf{1 0 0 . 0}$ & $\mathbf{1 0 0 . 0}$ & $\mathbf{1 0 0 . 0}$ & $\mathbf{1 0 0 . 0}$ & $\mathbf{1 0 0 . 0}$ \\
& Epileptic & 0.00 & 0.00 & 0.00 & 0.00 & 0.00 & 0.00 \\
\hline \multirow{2}{*}{ E } & Inter-ictal & 0.19 & 0.13 & 0.13 & 0.06 & 0.25 & 0.152 \\
& Epileptic & $\mathbf{9 9 . 8 1}$ & $\mathbf{9 9 . 8 7}$ & $\mathbf{9 9 . 8 7}$ & $\mathbf{9 9 . 9 4}$ & $\mathbf{9 9 . 7 5}$ & $\mathbf{9 9 . 8 5}$ \\
\hline
\end{tabular}


Table 5. Classification results of the five classifiers on all five data-sets of Case 3 (Back-Propagation Neural Network (BPNN), Support Vector Machine (SVM), Linear Discriminant Analysis (LDA), K-nearest neighbour (KNN), Decision Tree (DT), average value (Aver.)).

\begin{tabular}{cccccccc}
\hline Sets & Class & BPNN & SVM & LDA & KNN & DT & Aver. \\
\hline \multirow{4}{*}{ A } & Healthy & $\mathbf{9 9 . 2 8}$ & $\mathbf{9 9 . 7 5}$ & $\mathbf{9 6 . 4 8}$ & $\mathbf{9 1 . 7 1}$ & $\mathbf{9 9 . 6 2}$ & $\mathbf{9 7 . 3 7}$ \\
& Inter-ictal & 0.69 & 0.25 & 3.52 & 6.41 & 0.38 & 2.250 \\
& Epileptic & 0.03 & 0.00 & 0.00 & 1.88 & 0.00 & 0.382 \\
\hline \multirow{4}{*}{ B } & Healthy & $\mathbf{9 9 . 5 9}$ & $\mathbf{1 0 0 . 0}$ & $\mathbf{9 8 . 8 1}$ & $\mathbf{9 5 . 6 7}$ & $\mathbf{9 9 . 9 4}$ & $\mathbf{9 8 . 8 0}$ \\
& Inter-ictal & 0.35 & 0.00 & 1.19 & 2.51 & 0.06 & 0.822 \\
& Epileptic & 0.06 & 0.00 & 0.00 & 1.82 & 0.00 & 0.376 \\
\hline \multirow{2}{*}{ C } & Healthy & 1.16 & 0.13 & 0.50 & 0.13 & 1.88 & 0.760 \\
& Inter-ictal & $\mathbf{9 8 . 8 1}$ & $\mathbf{9 9 . 8 7}$ & $\mathbf{9 9 . 5 0}$ & $\mathbf{9 9 . 6 9}$ & $\mathbf{9 8 . 1 2}$ & $\mathbf{9 9 . 2 2}$ \\
& Epileptic & 0.03 & 0.00 & 0.00 & 0.19 & 0.00 & 0.044 \\
\hline \multirow{2}{*}{ D } & Healthy & 0.81 & 0.25 & 0.63 & 0.44 & 1.63 & 0.752 \\
& Inter-ictal & $\mathbf{9 9 . 1 5}$ & $\mathbf{9 9 . 7 5}$ & $\mathbf{9 9 . 3 7}$ & $\mathbf{9 9 . 5 6}$ & $\mathbf{9 8 . 3 7}$ & $\mathbf{9 9 . 2 4}$ \\
& Epileptic & 0.04 & 0.00 & 0.00 & 0.00 & 0.00 & 0.008 \\
\hline \multirow{2}{*}{ E } & Healthy & 0.16 & 0.00 & 0.06 & 0.06 & 1.01 & 0.258 \\
& Inter-ictal & 0.06 & 0.00 & 0.06 & 0.06 & 0.06 & 0.048 \\
& Epileptic & $\mathbf{9 9 . 7 8}$ & $\mathbf{1 0 0}$ & $\mathbf{9 9 . 8 8}$ & $\mathbf{9 9 . 8 8}$ & $\mathbf{9 8 . 9 3}$ & $\mathbf{9 9 . 6 9}$ \\
\hline
\end{tabular}

Table 6. Classification results of the five classifiers on all five data-sets of Case 4 (Back-Propagation Neural Network (BPNN), Support Vector Machine (SVM), Linear Discriminant Analysis (LDA), K-nearest neighbour (KNN), Decision Tree (DT), average value (Aver.)).

\begin{tabular}{cccccccc}
\hline Sets & Class & BPNN & SVM & LDA & KNN & DT & Aver. \\
\hline \multirow{4}{*}{ A } & Healthy-A & $\mathbf{8 9 . 3 8}$ & $\mathbf{9 7 . 0 5}$ & $\mathbf{8 2 . 9 8}$ & $\mathbf{9 2 . 0 9}$ & $\mathbf{9 3 . 2 8}$ & $\mathbf{9 0 . 0 6}$ \\
& Healthy-B & 9.36 & 2.95 & 15.64 & 4.21 & 6.22 & 7.680 \\
& Inter-ictal-C & 0.19 & 0.00 & 0.44 & 2.45 & 0.19 & 0.636 \\
& Inter-ictal-D & 0.94 & 0.00 & 0.94 & 0.82 & 0.31 & 0.602 \\
& Epileptic-E & 0.13 & 0.00 & 0.00 & 0.44 & 0.00 & 0.114 \\
\hline \multirow{6}{*}{ B } & Healthy-A & 11.68 & 3.45 & 12.63 & 4.15 & 5.53 & 7.488 \\
& Healthy-B & $\mathbf{8 7 . 2 5}$ & $\mathbf{9 6 . 5 5}$ & $\mathbf{8 7 . 0 0}$ & $\mathbf{9 4 . 9 1}$ & $\mathbf{9 4 . 2 8}$ & $\mathbf{9 2 . 0 0}$ \\
& Inter-ictal-C & 0.38 & 0.00 & 0.06 & 0.44 & 0.13 & 0.202 \\
& Inter-ictal-D & 0.57 & 0.00 & 0.31 & 0.19 & 0.06 & 0.226 \\
& Epileptic-E & 0.13 & 0.00 & 0.00 & 0.31 & 0.00 & 0.088 \\
\hline \multirow{4}{*}{ C } & Healthy-A & 0.63 & 0.06 & 0.38 & 0.00 & 0.19 & 0.252 \\
& Healthy-B & 0.32 & 0.06 & 0.00 & 0.00 & 0.31 & 0.138 \\
& Inter-ictal-C & $\mathbf{9 8 . 4 3}$ & $\mathbf{9 9 . 8 1}$ & $\mathbf{9 9 . 3 7}$ & $\mathbf{1 0 0 . 0}$ & $\mathbf{9 9 . 4 3}$ & $\mathbf{9 9 . 4 1}$ \\
& Inter-ictal-D & 0.57 & 0.06 & 0.25 & 0.00 & 0.06 & 0.188 \\
& Epileptic-E & 0.06 & 0.00 & 0.00 & 0.00 & 0.00 & 0.012 \\
\hline \multirow{4}{*}{ D } & Healthy-A & 0.50 & 0.31 & 0.63 & 0.06 & 0.88 & 0.476 \\
& Healthy-B & 0.19 & 0.00 & 0.00 & 0.00 & 0.38 & 0.114 \\
& Inter-ictal-C & 0.06 & 0.00 & 0.00 & 0.06 & 0.06 & 0.036 \\
& Inter-ictal-D & $\mathbf{9 9 . 1 8}$ & $\mathbf{9 9 . 6 9}$ & $\mathbf{9 9 . 3 7}$ & $\mathbf{9 9 . 8 7}$ & $\mathbf{9 8 . 6 8}$ & $\mathbf{9 9 . 3 6}$ \\
& Epileptic-E & 0.06 & 0.00 & 0.00 & 0.00 & 0.00 & 0.012 \\
\hline \multirow{6}{*}{ E } & Healthy-A & 0.06 & 0.06 & 0.06 & 0.00 & 0.06 & 0.048 \\
& Healthy-B & 0.25 & 0.00 & 0.00 & 0.00 & 0.25 & 0.100 \\
& Inter-ictal-C & 0.06 & 0.00 & 0.00 & 0.00 & 0.00 & 0.012 \\
& Inter-ictal-D & 0.06 & 0.00 & 0.00 & 0.00 & 0.00 & 0.012 \\
& Epileptic & $\mathbf{9 9 . 5 6}$ & $\mathbf{9 9 . 9 4}$ & $\mathbf{9 9 . 9 4}$ & $\mathbf{1 0 0 . 0}$ & $\mathbf{9 9 . 6 9}$ & $\mathbf{9 9 . 8 3}$ \\
\hline
\end{tabular}


Table 7. Average results obtained by the five classifiers after classification on variety train-to-test ratios (TTTRs) and variety numbers of Mel-frequency cepstrum coefficient (MFCC) features.

\begin{tabular}{|c|c|c|c|c|c|c|c|c|c|c|c|c|c|c|c|}
\hline \multirow{2}{*}{ Cases } & \multirow{2}{*}{ Set } & \multirow{2}{*}{ Class } & \multicolumn{9}{|c|}{ Train-to-Test Ratios (TTTRs) } & \multicolumn{4}{|c|}{ MFCC Features $\left(P F_{1}-P F_{4}\right)$} \\
\hline & & & 9:1 & $8: 2$ & $7: 3$ & 6:4 & $5: 5$ & 4:6 & 3:7 & $2: 8$ & $1: 9$ & $P F_{4}$ & $P F_{3-4}$ & $P F_{2-4}$ & $P F_{1-4}$ \\
\hline \multirow{10}{*}{ I } & \multirow{2}{*}{ A } & Healthy & 100.0 & 100.0 & 99.97 & 99.96 & 99.91 & 99.94 & 99.91 & 99.84 & 99.34 & 96.14 & 98.46 & 99.37 & 99.97 \\
\hline & & Epileptic & 0.00 & 0.00 & 0.03 & 0.04 & 0.09 & 0.06 & 0.09 & 0.16 & 0.66 & 3.86 & 1.54 & 0.63 & 0.03 \\
\hline & \multirow{2}{*}{ B } & Healthy & 100.0 & 100.0 & 99.97 & 99.91 & 99.87 & 99.78 & 99.87 & 99.84 & 99.47 & 93.66 & 7.11 & 98.90 & 99.97 \\
\hline & & Epileptic & 0.00 & 0.00 & 0.03 & 0.09 & 0.13 & 0.22 & 0.13 & 0.16 & 0.53 & 6.34 & 2.89 & 1.10 & 0.03 \\
\hline & \multirow{2}{*}{ C } & Healthy & 100.0 & 100.0 & 99.97 & 99.91 & 99.87 & 99.78 & 99.87 & $\begin{array}{l}99.84 \\
\end{array}$ & 99.47 & 93.66 & 7.11 & 98.90 & 99.97 \\
\hline & & Epileptic & 0.00 & 0.00 & 0.03 & 0.09 & 0.13 & 0.22 & 0.13 & 0.16 & 0.53 & 6.34 & 2.89 & 1.10 & 0.03 \\
\hline & \multirow{2}{*}{ D } & Healthy & 100.0 & 100.0 & 99.97 & 99.91 & 99.87 & 99.78 & 99.87 & 99.84 & 99.47 & 93.66 & 97.11 & 98.90 & 99.97 \\
\hline & & Epileptic & 0.00 & 0.00 & 0.03 & 0.09 & 0.13 & 0.22 & 0.13 & 0.16 & 0.53 & 6.34 & 2.89 & 1.10 & 0.03 \\
\hline & \multirow[b]{2}{*}{$\mathrm{E}$} & Healthy & 100.0 & 100.0 & 99.97 & 99.91 & 99.87 & 99.78 & 99.87 & 99.84 & 99.47 & 93.66 & 97.11 & 98.90 & 99.97 \\
\hline & & Epileptic & 0.00 & 0.00 & 0.03 & 0.09 & 0.13 & 0.22 & 0.13 & 0.16 & 0.53 & 6.34 & 2.89 & 1.10 & 0.03 \\
\hline \multirow{10}{*}{ II } & \multirow{2}{*}{ A } & Inter-ictal & 98.15 & 98.96 & 98.46 & 98.56 & 97.58 & 96.48 & 98.05 & 95.51 & 93.88 & 59.96 & 68.50 & 90.33 & 98.46 \\
\hline & & Epileptic & 1.85 & 1.04 & 1.54 & 1.44 & 2.42 & 3.52 & 1.95 & 4.49 & 6.12 & 40.04 & 31.44 & 9.67 & 1.54 \\
\hline & \multirow{2}{*}{ B } & Inter-ictal & 90.38 & 91.77 & 90.23 & 91.02 & 88.51 & 90.48 & 88.41 & 84.27 & 82.38 & 46.48 & 60.36 & 82.47 & 90.23 \\
\hline & & Epileptic & 9.62 & 8.23 & 9.77 & 8.98 & 11.49 & 9.52 & 11.59 & 15.73 & 17.62 & 53.52 & 39.64 & 17.53 & 9.77 \\
\hline & \multirow{2}{*}{ C } & Inter-ictal & 100.0 & 100.0 & 100.0 & 100.0 & 100.0 & 100.0 & 100.0 & 100.0 & 99.94 & 99.47 & 99.69 & 99.78 & 100.0 \\
\hline & & Epileptic & 0.00 & 0.00 & 0.00 & 0.00 & 0.00 & 0.00 & 0.00 & 0.00 & 0.06 & 0.53 & 0.31 & 0.22 & 0.00 \\
\hline & \multirow{2}{*}{$\mathrm{D}$} & Inter-ictal & 100.0 & 100.0 & 100.0 & 100.0 & 100.0 & 100.0 & 100.0 & 100.0 & 99.94 & 99.81 & 99.84 & 99.87 & 100.0 \\
\hline & & Epileptic & 0.00 & 0.00 & 0.00 & 0.00 & 0.00 & 0.00 & 0.00 & 0.00 & 0.06 & 0.19 & 0.16 & 0.13 & 0.00 \\
\hline & \multirow[b]{2}{*}{$\mathrm{E}$} & Inter-ictal & 0.12 & 0.19 & 0.19 & 0.31 & 0.19 & 0.82 & 0.44 & 0.57 & 1.00 & 1.57 & 0.72 & 0.50 & 0.19 \\
\hline & & Epileptic & 99.88 & 99.81 & 99.81 & 99.69 & 99.81 & 99.18 & 99.56 & 99.43 & 99.00 & 98.43 & 99.28 & 99.50 & 99.81 \\
\hline \multirow{15}{*}{ III } & \multirow{3}{*}{ A } & $\mathrm{H}$ & 98.62 & 99.03 & 99.28 & 98.59 & 97.99 & 97.49 & 96.33 & 96.04 & 90.20 & 89.89 & 95.79 & 97.90 & 99.28 \\
\hline & & Inter-ictal & 1.35 & 0.94 & 0.69 & 1.32 & 2.01 & 2.42 & 3.58 & 3.61 & 7.19 & 4.46 & 2.544 & 1.288 & 0.69 \\
\hline & & Epileptic & 0.03 & 0.03 & 0.03 & 0.09 & 0.06 & 0.09 & 0.47 & 0.41 & 0.19 & 3.08 & 1.947 & 0.691 & 0.03 \\
\hline & \multirow{3}{*}{ B } & Healthy & 99.59 & 99.69 & 99.59 & 99.31 & 99.03 & 99.00 & 98.46 & 97.24 & 96.04 & 88.25 & 95.23 & 98.27 & 99.59 \\
\hline & & Inter-ictal & 0.38 & 0.25 & 0.3 & 0.53 & 0.88 & 08 & 1.41 & 2.07 & 28 & 2.95 & 2.136 & 1.099 & 0.35 \\
\hline & & Epileptic & 0.03 & 0.06 & 0.06 & 0.13 & 0.19 & 0.06 & 0.09 & 0.60 & 0.38 & 6.66 & 3.109 & 0.754 & 0.06 \\
\hline & \multirow{3}{*}{ C } & Healthy & 0.88 & 0.4 & 1.1 & 1.01 & 1.32 & 1.48 & 1.10 & 1.7 & 2.3 & 5.28 & 2.481 & 1.413 & 1.16 \\
\hline & & Inter-ictal & 99.12 & 99.53 & 98.81 & 99.00 & 98.68 & 98.46 & 98.90 & 98.12 & 96.86 & 90.58 & 97.39 & 98.62 & 98.81 \\
\hline & & Epileptic & 0.00 & 0.03 & 0.03 & 0.09 & 0.06 & 0.06 & 0.06 & 0.13 & 0.25 & 0.31 & 0.188 & 0.188 & 0.03 \\
\hline & \multirow{3}{*}{$\mathrm{D}$} & Healthy & 0.50 & 0.57 & 0.81 & 0.85 & 1.60 & 1.32 & 1.88 & 3.20 & 1.98 & 2.01 & 1.539 & 1.382 & 0.81 \\
\hline & & Inter-ictal & 99.50 & 99.37 & 99.15 & 99.15 & 98.40 & 98.71 & 98.08 & 98.02 & 97.14 & 96.98 & 98.27 & 98.62 & 99.15 \\
\hline & & Epileptic & 0.00 & 0.06 & 0.04 & 0.09 & 0.03 & 0.06 & 0.09 & 0.29 & 0.25 & 0.44 & 0.126 & 0.063 & 0.04 \\
\hline & \multirow{3}{*}{ E } & 1. & 0. & 0. & 0. & 0. & 0. & 0 & 0. & 1. & 1. & 11.81 & 5.8 & 0.817 & 0.16 \\
\hline & & Inter-ictal & 0.25 & 0.13 & 0.0 & 0.38 & 0.16 & 0.41 & 0.47 & 0.63 & 1.44 & 0.38 & 0.157 & 0.157 & 0.06 \\
\hline & & Epileptic & 99.53 & 99.64 & 99.78 & 99.84 & 99.56 & 99.65 & 99.50 & 98.59 & 98.93 & 81.47 & 93.97 & 97.74 & 99.78 \\
\hline
\end{tabular}


Table 7. Cont.

\begin{tabular}{|c|c|c|c|c|c|c|c|c|c|c|c|c|c|c|c|}
\hline \multirow{2}{*}{ Cases } & \multirow{2}{*}{ Set } & \multirow{2}{*}{ Class } & \multicolumn{9}{|c|}{ Train-to-Test Ratios (TTTRs) } & \multicolumn{4}{|c|}{ MFCC Features $\left(P F_{1}-P F_{4}\right)$} \\
\hline & & & 9:1 & $8: 2$ & $7: 3$ & $6: 4$ & 5:5 & $4: 6$ & 3:7 & $2: 8$ & 1:9 & $\mathrm{PF}_{4}$ & $P F_{3-4}$ & $P F_{2-4}$ & $P F_{1-4}$ \\
\hline \multirow{25}{*}{ IV } & \multirow{5}{*}{ A } & Healthy-A & 90.57 & 89.64 & 89.38 & 85.11 & 80.31 & 78.71 & 76.27 & 72.33 & 50.69 & 41.24 & 53.64 & 70.32 & 89.38 \\
\hline & & & 9.08 & 7.16 & 9.36 & 11.78 & 13.38 & 17.43 & 19.50 & 15.42 & 17.18 & 16.11 & 18.28 & 19.54 & 9.36 \\
\hline & & Inter-ictal-C & 0.13 & 0.38 & 0.19 & 0.41 & 0.31 & 0.82 & 1.04 & 1.41 & 2.98 & 4.08 & 1.51 & 0.60 & 0.19 \\
\hline & & Inter-ictal-D & 0.22 & 0.28 & 0.94 & 0.47 & 0.44 & 1.44 & 1.04 & 1.44 & 298 & 88 & 0.50 & 38 & 0.94 \\
\hline & & Epileptic-E & 0.00 & 0.06 & 0.13 & 0.13 & 0.35 & 0.13 & 0.44 & 0.13 & 0.97 & 3.89 & 1.76 & 0.47 & 0.13 \\
\hline & \multirow{5}{*}{ B } & 1. & 30 & . & 1 & 16.08 & 5 & 1 & 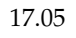 & 5 & 21.86 & 0 & . & 25.50 & 1.68 \\
\hline & & Healthy & 90.42 & 86.65 & 87.25 & 84.01 & 83.20 & 82.88 & 82.00 & 6.61 & 52.42 & 28.02 & & 60.33 & 87.25 \\
\hline & & Inter-ictal-C & 0.03 & 0.13 & & 0.13 & & & & 0.94 & & & & & 0.38 \\
\hline & & & 0.04 & 0.0 & 0.5 & 0.3 & 0. & 0. & 0.6 & & & & & & 0.57 \\
\hline & & Epileptic-E & 0.03 & 0.13 & 0.13 & 0.09 & 0.31 & 0.19 & 0.41 & 0.19 & 0.91 & 7.44 & 2.70 & 1.88 & 0.13 \\
\hline & \multirow{5}{*}{ C } & Healthy-A & 60 & 0.22 & 0.63 & 7.82 & 0. & 2.04 & 17 & 2. & 4.5 & 0.8 & 0.2 & 0.38 & 0.63 \\
\hline & & Healt & 0.06 & 0.16 & 0.32 & 0.82 & 0.35 & 0.88 & 1.44 & 1.73 & & 38 & 0.06 & 0.41 & 0.32 \\
\hline & & Inter-ictal-C & 98.12 & 99.47 & 98.43 & 97.58 & 99.18 & 98.24 & 98.74 & 94.44 & 58.76 & 90.64 & 97.68 & 98.74 & 98.43 \\
\hline & & Inter-ictal-D & 0.09 & 0.13 & 0.57 & 0.22 & 0.09 & 1.44 & 0.82 & 0.35 & 0.66 & 0.06 & 0.00 & 0.03 & 0.57 \\
\hline & & Epileptic-E & 0.03 & 0.06 & 0.06 & 0.06 & 0.09 & 0.03 & 0.06 & 0.03 & 2.64 & 0.41 & 0.06 & 0.03 & 0.06 \\
\hline & \multirow{5}{*}{ D } & Healthy-A & 1.1 & 0.69 & 0.5 & 0.7 & 1.0 & 7.67 & 2.1 & 3. & 4.52 & 0.6 & 0.3 & 0. & 0.50 \\
\hline & & & 0. & & & & & 0 & & & & & & & 0.19 \\
\hline & & Inter-ictal-C & 0.03 & 0.06 & 0.0 & 0.1 & & 0.22 & 0.09 & 0.25 & 1.48 & 0.22 & 0.00 & 0.03 & 0.06 \\
\hline & & Inter- & 98.74 & 97.93 & 99.18 & 98.96 & 99.21 & 97.80 & 98.27 & 90.70 & 67.71 & 96.36 & 6 & 98.68 & 99.18 \\
\hline & & Epileptic-E & 0.00 & 0.03 & 0.06 & 0.06 & 0.03 & 0.03 & 0.47 & 0.63 & 0.44 & 0.25 & 0.00 & 0.03 & 0.06 \\
\hline & \multirow{5}{*}{ E } & & & 0 & 0.06 & & & 0.75 & & 1. & & & 0 & & 6 \\
\hline & & & 0. & 0. & 0.2 & & & 0.3 & 0.8 & & & & 1.7 & & 0.25 \\
\hline & & Inter & 0.00 & 0.09 & 0.0 & 0.0 & 0.03 & 0.09 & 0.09 & 0.1 & 1.4 & 0.60 & 0.00 & 0.03 & 0.06 \\
\hline & & & 0.00 & 0.03 & 0.06 & 0.03 & 0.03 & 0.79 & 0.16 & 0.3 & 0.63 & 0.13 & 0.00 & 0.03 & 0.06 \\
\hline & & Epileptic-E & 99.85 & 99.78 & 99.56 & 99.81 & 99.69 & 99.75 & 94.72 & 99.37 & 61.90 & 78.14 & 91.52 & 96.77 & 99.56 \\
\hline
\end{tabular}


From the results in Tables 3-6, an interesting phenomenon revealed that datasets $C$ and D always contained some healthy EEG components, which lowered the classification accuracy in Tables 3,5 and 6, and increased the classification accuracy in Table 4. One possible explanation is that the structures of brain cells have a few common features, which imparts the EEG segment with some commonalities with healthy EEG segments when the motivation signals through these neurons. Similarly, the epileptic EEG segments also have some common features to those of healthy EEG segments, as shown in Tables 3, 5 and 6.

In order to eliminate the influence of the number of training samples on the recognition result, the majority of the results in Tables 2-6 were obtained using 7:3 as the TTTR. In order to test the robustness of the computational performance with varying TTTRs, Table 7 provides the recognition results obtained with the BPNN classifier. As can be seen in Table 7, the results obtained by this proposed method do not significantly rely on a high TTTR.

\subsection{Performance Analysis}

As presented in Tables 2-8, regardless of the case, all the classifiers could be used to provide high classification accuracy. On one hand, the proposed method can be employed to realize a maximal classification accuracy at 100\% in Cases 1 and 2. On the other hand, it still provides a high classification accuracy of $99.98 \%$ in Case 3, as well as a maximum accuracy of $98.35 \%$ in Case 4 , as shown in Table 2 . Furthermore, combining all the features from PF1 to PF4 resulted in better performance than the others in all cases, as shown in Table 7. One plausible explanation is that the information transmission must be distributed to prevent the effects of noise, transmission channel blocking, etc. Another possible explanation is that PF1 contains more significant features than the others, such as healthy versus seizure, seizure-free versus seizure, healthy versus seizure-free versus seizure, etc. Table 8 presents the confusion matrix corresponding to the four cases.

Generally, the performance of this automated seizure detection method can be analyzed using three criteria:

- Detection sensitivity (SEN). This method successfully detected 1562, 1549, 1583, and 1406 of 1592 healthy events, which amount to detection sensitivities of $98.12 \%, 97.30 \%, 99.43 \%$, and $88.32 \%$, respectively. The detection sensitivity can be as high as $100 \%$ and as low as $99.94 \%$.

- False positives (specificity; SPE). This method successfully detected 1589, 1589, 1588, and 1585 of 1592 seizure events, which amount to detection sensitivities of $99.81 \%, 99.81 \%, 99.75 \%$, and $99.56 \%$, respectively. A good seizure detector must achieve maximum seizure detection sensitivity while minimizing false detections. Even with high sensitivity, a large amount of false detections can be problematic for the patient and medical staff in real-life applications. In this study, the false positives were as low as $0.00 \%$ and as high as $9.36 \%$.

- Classification accuracy (CA) is considered as a statistical measure by which the performance of a classification algorithm can be evaluated. It is computed by dividing the number of correctly classified samples by the total number of samples. This method was successfully used to obtain accuracies as high as $99.91 \%$ and as low as $93.41 \%$.

Therefore, strong universality has been proven and can be considered as the greatest aspect of this method. Furthermore, the results of these four cases illustrate the robustness of this method, which accordingly has the potential to be applied to clinical cases with a high classification accuracy. 
Table 8. The confusion matrix corresponding to the four cases for performance analysis (Back-Propagation Neural Network (BPNN)).

\begin{tabular}{|c|c|c|c|c|c|c|c|c|c|c|c|c|c|c|c|}
\hline Cases & Set & Class & $\bar{a}$ & $\mathbf{b}$ & $\mathrm{c}$ & $\mathrm{d}$ & AC & TP & FP & TN & FN & $\mathbf{P}$ & SEN & SPE & $\mathrm{CA}$ \\
\hline \multirow{10}{*}{ I } & \multirow{2}{*}{ A } & Healthy & 1592 & 0 & 3 & 1589 & 99.91 & 99.81 & 0.00 & 100.0 & 0.19 & 100.0 & 99.81 & 100.0 & 99.91 \\
\hline & & Epileptic & 3 & 1589 & 0 & 1592 & 50.09 & 100.0 & 99.81 & 0.19 & 0.00 & 50.05 & 100.0 & 0.19 & 50.10 \\
\hline & \multirow{2}{*}{ B } & Healthy & 1592 & 0 & 3 & 1589 & 99.91 & 99.81 & 0.00 & 100.0 & 0.19 & 100.0 & 99.81 & 100.0 & 99.91 \\
\hline & & Epileptic & 3 & 1589 & 0 & 1592 & 50.09 & 100.0 & 99.81 & 0.19 & 0.00 & 50.05 & 100.0 & 0.19 & 50.10 \\
\hline & \multirow{2}{*}{ C } & Healthy & 1487 & 105 & 3 & 1589 & 96.61 & 99.81 & 6.60 & 93.40 & 0.19 & 96.07 & 99.81 & 93.40 & 96.61 \\
\hline & & Epileptic & 3 & 1589 & 0 & 1592 & 50.09 & 100.0 & 99.81 & 0.19 & 0.00 & 50.05 & 100.0 & 0.19 & 50.10 \\
\hline & \multirow{2}{*}{$\mathrm{D}$} & Healthy & 1575 & 17 & 3 & 1589 & 99.37 & 99.81 & 1.07 & 98.93 & 0.19 & 98.94 & 99.81 & 98.93 & 99.37 \\
\hline & & Epileptic & 3 & 1589 & 0 & 1592 & 50.09 & 100.0 & 99.81 & 0.19 & 0.00 & 50.05 & 100.0 & 0.19 & 50.10 \\
\hline & \multirow[b]{2}{*}{ E } & Healthy & 0 & 1592 & 3 & 1589 & 49.91 & 99.81 & 100.0 & 0.00 & 0.19 & 50.05 & 99.81 & 0.00 & 49.91 \\
\hline & & Epileptic & 1589 & 3 & 0 & 1592 & 99.91 & 100.0 & 0.19 & 99.81 & 0.00 & 99.81 & 100.0 & 99.81 & 99.91 \\
\hline \multirow{10}{*}{ II } & \multirow{2}{*}{ A } & Inter-ictal & 1578 & 14 & 3 & 1589 & 99.12 & 99.81 & 0.88 & 99.12 & 0.19 & 99.13 & 99.81 & 99.12 & 99.47 \\
\hline & & Epileptic & 3 & 1589 & 91 & 1501 & 47.24 & 94.28 & 99.81 & 0.19 & 5.72 & 48.58 & 94.28 & 0.19 & 47.24 \\
\hline & \multirow{2}{*}{ B } & Inter-ictal & 1435 & 157 & 3 & 1589 & 94.97 & 99.81 & 9.86 & 90.14 & 0.19 & 91.01 & 99.81 & 90.14 & 94.98 \\
\hline & & Epileptic & 3 & 1589 & 91 & 1501 & 47.24 & 94.28 & 99.81 & 0.19 & 5.72 & 48.58 & 94.28 & 0.19 & 47.24 \\
\hline & \multirow{2}{*}{ C } & Inter-ictal & 1592 & 0 & 3 & 1589 & 99.91 & 99.81 & 0.00 & 100.0 & 0.19 & 100.0 & 99.81 & 100.0 & 99.91 \\
\hline & & Epileptic & 3 & 1589 & 91 & 1501 & 47.24 & 94.28 & 99.81 & 0.19 & 5.78 & 48.58 & 94.28 & 0.19 & 47.24 \\
\hline & \multirow{2}{*}{$\mathrm{D}$} & Inter-ictal & 1592 & 0 & 3 & 1589 & 99.91 & 99.81 & 0.00 & 100.0 & 0.19 & 100.0 & 99.81 & 100.0 & 99.91 \\
\hline & & Epileptic & 3 & 1589 & 91 & 1501 & 47.24 & 94.28 & 99.81 & 0.19 & 5.72 & 48.58 & 94.28 & 0.19 & 47.24 \\
\hline & \multirow{2}{*}{ E } & Inter-ictal & 3 & 1589 & $\mathrm{~J}$ & 1589 & 50.00 & 99.81 & 99.81 & 0.19 & 0.19 & 50.00 & 99.81 & 0.19 & 50.00 \\
\hline & & Epileptic & 1589 & 3 & 91 & 1501 & 97.05 & 94.28 & 0.19 & 99.81 & 7.52 & 99.80 & 94.28 & 99.81 & 99.78 \\
\hline \multirow{15}{*}{ III } & \multirow{3}{*}{ A } & althy & 1579 & 13 & 4 & 1588 & 99.37 & 99.75 & 0.82 & 99.18 & 0.25 & 99.19 & 99.75 & 99.18 & 99.47 \\
\hline & & Inter-ictal & 11 & 1581 & 16 & 1576 & 49.84 & 98.99 & 99.31 & 0.69 & 1.01 & 49.92 & 98.99 & 0.69 & 49.84 \\
\hline & & Epileptic & 2 & 1590 & 2 & 1590 & 50.00 & 99.87 & 99.87 & 0.13 & 0.13 & 50.00 & 99.87 & 0.13 & 50.00 \\
\hline & \multirow{3}{*}{ B } & Healtby & 1587 & 5 & 4 & 1588 & 99.72 & 99.75 & 0.31 & 99.69 & 0.25 & 99.69 & 99.75 & 99.69 & 99.72 \\
\hline & & Inter-ictal & 4 & 1588 & 16 & 1576 & 49.62 & 98.99 & 99.72 & 0.28 & 1.01 & 49.81 & 98.99 & 0.28 & 49.64 \\
\hline & & Epileptic & 1 & 1591 & 2 & 1590 & 49.97 & 99.87 & 99.94 & 0.06 & 0.13 & 49.94 & 99.87 & 0.06 & 49.97 \\
\hline & \multirow{3}{*}{ C } & Healthy & 1 & 1573 & 16 & 1576 & 50.09 & 98.99 & 98.8 & 1.19 & 1.01 & 50.05 & 98.99 & 1.1 & 50.09 \\
\hline & & Inter-ictal & 1572 & 20 & 4 & 1588 & 99.06 & 99.75 & 1.26 & 98.74 & 0.25 & 98.69 & 99.75 & 98.74 & 99.25 \\
\hline & & Epileptic & 1 & 1591 & 2 & 1590 & 49.97 & 99.87 & 99.94 & 0.06 & 0.13 & 49.94 & 99.87 & 0.06 & 49.97 \\
\hline & \multirow{3}{*}{$\mathrm{D}$} & ealt the & 12 & 1580 & 16 & 1576 & 49.87 & 98.5 & 99.25 & 0.7 & 1.01 & 49.94 & 98.99 & 0.75 & 49.87 \\
\hline & & Inter-ictal & 1579 & 13 & 2 & 1590 & 99.37 & 99.87 & 0.82 & 99.18 & 0.13 & 99.19 & 99.87 & 99.18 & 99.53 \\
\hline & & Epileptic & 1 & 1591 & 2 & 1590 & 49.97 & 99.87 & 99.94 & 0.06 & 0.13 & 49.94 & 99.87 & 0.06 & 49.97 \\
\hline & \multirow{3}{*}{$\mathrm{E}$} & 1 & 2 & 1590 & 9 & 1583 & 49.78 & 99.43 & 99.87 & 0.13 & 0.57 & 49.89 & 99.43 & 0.13 & 49.78 \\
\hline & & Inter-ictal & 2 & 1590 & 1 & 1591 & 50.03 & 99.94 & 99.87 & 0.13 & 0.06 & 50.06 & 99.94 & 0.13 & 50.04 \\
\hline & & Epileptic & 1588 & 4 & 2 & 1590 & 99.81 & 99.87 & 0.25 & 99.75 & 0.13 & 99.75 & 99.87 & 99.75 & 99.81 \\
\hline
\end{tabular}


Table 8. Cont.

\begin{tabular}{|c|c|c|c|c|c|c|c|c|c|c|c|c|c|c|c|}
\hline Cases & Set & Class & $\mathbf{a}$ & $\mathbf{b}$ & c & $d$ & AC & TP & FP & TN & FN & $\mathbf{P}$ & SEN & SPE & $\mathrm{CA}$ \\
\hline \multirow{25}{*}{ IV } & \multirow{5}{*}{ A } & Healthy-A & 1423 & 169 & 7 & 1585 & 94.47 & 99.56 & 0.11 & 99.89 & 0.44 & 90.36 & 99.56 & 99.89 & 99.73 \\
\hline & & Healthy-B & 149 & 1443 & 2 & 1590 & 54.62 & 99.87 & 90.64 & 9.36 & 0.13 & 52.42 & 99.87 & 9.36 & 54.62 \\
\hline & & Inter-ictal-C & 3 & 1589 & 10 & 1582 & 49.78 & 99.37 & 99.81 & 0.19 & 0.63 & 49.89 & 99.37 & 0.19 & 49.78 \\
\hline & & Inter-ictal-D & 15 & 1577 & 8 & 1584 & 50.22 & 99.50 & 99.06 & 0.94 & 0.50 & 50.11 & 99.50 & 0.94 & 50.22 \\
\hline & & Epileptic-E & 2 & 1590 & 1 & 1591 & 50.03 & 99.94 & 99.87 & 0.13 & 0.06 & 50.06 & 99.94 & 0.13 & 50.04 \\
\hline & \multirow{5}{*}{ B } & Healthy-A & 186 & 1406 & 149 & 1443 & 51.16 & 90.64 & 88.32 & 11.68 & 9.36 & 50.65 & 90.64 & 11.68 & 51.16 \\
\hline & & Healthy-B & 1389 & 203 & 7 & 1585 & 93.41 & 99.56 & 12.75 & 87.25 & 0.44 & 88.65 & 99.56 & 87.25 & 93.41 \\
\hline & & Inter-ictal-C & 6 & 1586 & 5 & 1587 & 50.03 & 99.69 & 99.62 & 0.38 & 0.31 & 50.02 & 99.69 & 0.38 & 50.04 \\
\hline & & Inter-ictal-D & 9 & 1583 & 3 & 1589 & 50.19 & 99.81 & 99.43 & 0.57 & 0.19 & 50.09 & 99.81 & 0.57 & 50.19 \\
\hline & & Epileptic-E & 2 & 1590 & 4 & 1588 & 49.94 & 99.75 & 99.87 & 0.13 & 0.25 & 49.97 & 99.75 & 0.13 & 49.94 \\
\hline & \multirow{5}{*}{ C } & Healthy-A & 10 & 1582 & 3 & 1589 & 50.22 & 99.81 & 99.37 & 0.63 & 0.19 & 50.11 & 99.81 & 0.63 & 50.22 \\
\hline & & Healthy-B & 5 & 1587 & 6 & 1586 & 49.97 & 99.62 & 99.69 & 0.31 & 0.38 & 49.98 & 99.62 & 0.31 & 49.97 \\
\hline & & Inter-ictal-C & 1567 & 25 & 7 & 1585 & 98.99 & 99.56 & 1.57 & 98.43 & 0.44 & 98.45 & 99.56 & 98.43 & 99.03 \\
\hline & & Inter-ictal-D & 9 & 1583 & 1 & 1591 & 50.25 & 99.94 & 99.43 & 0.57 & 0.06 & 50.13 & 99.94 & 0.57 & 50.26 \\
\hline & & Epileptic-E & 1 & 1591 & 1 & 1591 & 50.00 & 99.94 & 99.94 & 0.06 & 0.06 & 50.00 & 99.94 & 0.06 & 50.00 \\
\hline & \multirow{5}{*}{ D } & Healthy-A & 8 & 1584 & 15 & 1577 & 49.78 & 99.06 & 99.50 & 0.50 & 0.94 & 49.89 & 99.06 & 0.50 & 49.78 \\
\hline & & Healthy-B & 3 & 1589 & 9 & 1583 & 49.81 & 99.43 & 99.81 & 0.19 & 0.57 & 49.91 & 99.43 & 0.19 & 49.81 \\
\hline & & Inter-ictal-C & 1 & 1591 & 9 & 1583 & 49.75 & 99.43 & 99.94 & 0.06 & 0.57 & 49.87 & 99.43 & 0.06 & 49.75 \\
\hline & & Inter-ictal-D & 1579 & 13 & 7 & 1585 & 99.37 & 99.56 & 0.82 & 99.18 & 0.44 & 99.19 & 99.56 & 99.18 & 99.37 \\
\hline & & Epileptic-E & 1 & 1591 & 1 & 1591 & 50.00 & 99.94 & 99.94 & 0.06 & 0.06 & 50.00 & 99.94 & 0.06 & 50.00 \\
\hline & \multirow{5}{*}{ E } & Healthy-A & 1 & 1591 & 2 & 1590 & 49.97 & 99.87 & 99.94 & 0.06 & 0.13 & 49.98 & 99.87 & 0.06 & 49.97 \\
\hline & & Healthy-B & 4 & 1588 & 2 & 1590 & 50.06 & 99.87 & 99.75 & 0.25 & 0.13 & 50.03 & 99.87 & 0.25 & 50.06 \\
\hline & & Inter-ictal-C & 1 & 1591 & 1 & 1591 & 50.00 & 99.94 & 99.94 & 0.06 & 0.06 & 50.00 & 99.94 & 0.06 & 50.00 \\
\hline & & Inter-ictal-D & 1 & 1591 & 1 & 1591 & 50.00 & 99.94 & 99.94 & 0.06 & 0.06 & 50.00 & 99.94 & 0.06 & 50.00 \\
\hline & & Epileptic-E & 1585 & 7 & 1 & 1591 & 99.75 & 99.94 & 0.44 & 99.56 & 0.06 & 99.56 & 99.94 & 99.56 & 99.75 \\
\hline
\end{tabular}




\subsection{Comparison with Related State-of-the-Art Work}

A comparison between this automatic epileptic seizure detection method and the existing state-of-the-art works from 2014 to 2017 is provided in Table 9. The previously reported methods $[8,9,11,16,26]$ were tested with the identified EEG segment, thus making the comparison more feasible and providing additional perspective. All the recognition results for these methods $[8,9,11,16,26]$ were copied from their reports. The recognition results of this method include the averages of the five classifiers by setting the TTTR to 7:3.

Table 9. The comparison between this method and other methods using the same database.

\begin{tabular}{|c|c|c|c|c|}
\hline Year & Authors & Methods & Tasks & Aver. \\
\hline 2014 & Y. Kumar et al. [14] & $\begin{array}{l}\text { Discrete wavelet transform (DWT) } \\
\text { Fuzzy approximate entropy (fApEn) } \\
\text { Support vector machines (SVMs) }\end{array}$ & $\begin{array}{c}\text { A-E, B-E, C-E, } \\
\text { D-E, ACD-E, } \\
\text { BCD-E, ABCD-E }\end{array}$ & $\begin{array}{c}\text { 100.0, 100.0, } 99.60 \\
95.85,98.15 \\
98.22,97.38\end{array}$ \\
\hline 2015 & K. Samiee et al. [7] & rational functions (RFs) & $\begin{array}{c}\text { A-E, B-E, C-E, } \\
\text { D-E, A, B, C, D-E }\end{array}$ & $\begin{array}{c}99.80,99.30,98.50, \\
94.90,98.10\end{array}$ \\
\hline 2016 & D. Li et al. [11] & $\begin{array}{l}\text { Discrete Wavelet Transforms (DWT) } \\
\text { Phase Space Reconstruction (PSR) } \\
\text { Singular values of covariance matrix }\end{array}$ & $\begin{array}{l}\text { A-E, B-E, C-E, } \\
\text { D-E, ABCD-E, } \\
\text { AB-CDE, CD-E }\end{array}$ & $\begin{array}{c}99.97,99.92,99.82 \\
99.76,99.38 \\
99.53,98.89\end{array}$ \\
\hline \multirow[b]{2}{*}{2017} & T. Zhang et al. [22] & $\begin{array}{l}\text { local mean decomposition (LMD) } \\
\text { BPNN, KNN, LDA, SVM, etc. }\end{array}$ & $\begin{array}{l}\text { A-E, D-E, ABCD-E, } \\
\text { A-D-E, AB-CD-E }\end{array}$ & $\begin{array}{c}100.0,98.10,98.87 \\
98.47,98.40\end{array}$ \\
\hline & M. Mursalin et al. [26] & $\begin{array}{c}\text { Discrete Wavelet transformation (DWT) } \\
\text { Correlation-based Feature Selection (CFS) } \\
\text { Improved Correlation-based Feature } \\
\text { Random Forest (RF) }\end{array}$ & $\begin{array}{l}\text { A-E, B-E, C-E, } \\
\text { D-E, ACD-E, } \\
\text { BCD-E, CD-E, } \\
\text { ABCD-E }\end{array}$ & $\begin{array}{c}100.0,98.00,99.00 \\
98.50,98.50 \\
97.50,98.67 \\
97.40\end{array}$ \\
\hline 2018 & This paper & $\begin{array}{l}\text { The novel hypothesis } \\
\text { LMD, MFCC } \\
\text { BPNN, KNN, LDA } \\
\text { SVM, DT }\end{array}$ & $\begin{array}{l}\text { A-E, B-E, C-E, } \\
\text { D-E, ABCD-E, } \\
\text { AB-CD-E, CD-E, } \\
\text { A-B-C-D-E }\end{array}$ & $\begin{array}{c}100.0,100.0,99.86 \\
99.78,99.46 \\
97.85,99.83 \\
97.43\end{array}$ \\
\hline
\end{tabular}

Taking dataset $\mathrm{A}$ versus dataset $\mathrm{E}$ and dataset $\mathrm{B}$ versus dataset $\mathrm{E}$ as two examples, the maximum recognition accuracy of the presented method was as high as $100 \%$, whereas some others also produced results as high as $100 \%$. From Table 9, the recognition accuracy obtained in classifying healthy and seizure classes (dataset A versus dataset E and dataset B versus dataset E) was as high as $100 \%$. The seizure-free and seizure classes (dataset $C$ versus dataset $E$ and dataset D versus dataset $E$ ) obtained by this proposed method was at least as high as $99.83 \%$, but the existing methods could only reach a maximum accuracy of $99.82 \%$.

When considering all five of the EEG datasets, the classification task problems were datasets $A, B, C$, and D, versus dataset $E$; datasets $A$ and $B$ versus dataset $C$ and dataset $D$ versus dataset $\mathrm{E}$; and dataset $A$ versus dataset $B$ versus dataset $C$ versus dataset $D$ versus dataset E. Fortunately, this proposed method can be utilized to achieve high accuracies, of $99.46 \%, 97.85 \%$, and $97.43 \%$, respectively, and the other methods were not as accurate.

\section{Conclusions and Future Works}

The goal of this paper was to attempt to solve the nonlinear and non-stationary problems of analyzing EEG signals to achieve recognition of healthy, epileptic seizure-free, and epileptic seizure patients. For this reason, an automated epileptic seizure detection method was constructed. The idea was sourced from a novel and reasonable hypothesis that mimics the generation mechanism of EEG signals to some sense.

From the novel hypothesis, the characteristics of EEG signals depended on the construction of the brain cells and these EEG signals involved the macroscopic performance of a large number of neurons. This means that the EEG signals should be first decomposed into several subcomponents that describe the different states of brain, from low frequency to high frequency, which can be employed to 
demonstrate the active or non-active areas of neurons. A local mean decomposition algorithm was able to attain this goal, and can be considered as the solution to the non-stationary problem.

The problem then becomes how to separate these subcomponents from active or non-active areas of neurons into the feature vectors, which can be used to achieve EEG segment recognition. The Mel-frequency cepstrum coefficient (MFCC) was used to achieve the deconvolution calculation to separate these various subcomponents, and can be considered as the solution to the nonlinear problem.

In future works, the suitable length of the window overlap to build a new EEG segment should be studied by determining the impacts of the length of the signal acquisition changes on the amount of information. Secondly, we hope to evaluate our proposed method for automatically analyzing long recording EEG signals. Thirdly, we will apply this method to solving the problem of classifying multichannel sleep EEG signals and the recognition of different individual behaviors using EEG signals.

Furthermore, ultrasound and optoacoustics, applied in epileptic seizure detection, can be considered as the recent advances in this field, which have been reported in mainstream journals [17-24]. Therefore, these two methods will be the research areas that require most of our attention and practical action.

Author Contributions: H.R. designed the overall framework of this paper, built the simulation model and completed the manuscript. J.Q. and Y.C. modified and refined the manuscript. Q.T. and L.H. conducted data analysis.

Funding: This research received no external funding.

Acknowledgments: This work was supported by the National Natural Science Foundation of China, through Grant 61633005, Grant 61673076 and Grant 61773080, and by the Project No.106112016CDJXZ238826 and 2018CDYISY0055 supported by the Fundamental Research Funds for the Central Universities, and funded by the Natural Science Foundation of Chongqing City, China(cstc2016jcyjA0504), and funded by the Natural Science Research Project of the Higher Education Institutions of Jiangsu Province China (18KJB510006).

Conflicts of Interest: The authors declare no conflict of interest.

\section{References}

1. Acharya, U.R.; Fujita, H.; Sudarshan, V.K.; Bhat, S.; Koh, J.E.W. Application of Entropies for automated diagnosis of epilepsy using EEG signals: A review. Knowl. Based Syst. 2015, 88, 85-96. [CrossRef]

2. Nunes, T.M.; Coelho, A.L.V.; Lima, C.A.M.; Papa, J.P.; Albuquerque, V.H.C.D. EEG signal classification for epilepsy diagnosis via optimum path forest-A systematic assessment. Neurocomputing 2014, 136, 103-123. [CrossRef]

3. Ramgopal, S.; Thome-Souza, S.; Jachson, M.; Kadish, N.E.; Sánchez, F.I.; Klehm, J.; Bosl, W.; Reinsberger, C.; Schachter, S.; Loddenkemper, T. Seizure detection, seizure prediction, and closed-loop warning systems in epilepsy. Epilepsy Behav. 2014, 37, 291-307. [CrossRef] [PubMed]

4. Acharya, U.R.; Stree, S.V.; Swapna, G.; Martis, R.J.; Suri, J.S. Automated EEG analysis of epilepsy: A review. Knowl. Based Syst. 2013, 45, 147-165. [CrossRef]

5. Ren, H.; Qu, J.F.; Chai, Y.; Tang, Q.; Zhou, Y. An adaptive unsupervised classification of seizure method based on LMD-MSSE with EEG signals. Biomed. Res. India 2017, 28, 3151-3158.

6. Swami, P.; Gandhi, T.K.; Panigrahi, B.K.; Tripathi, M.; Anand, S. A novel robust diagnostic model to detect seizures in electroencephalography. Expert Syst. Appl. 2016, 56, 116-130. [CrossRef]

7. Parvez, M.Z.; Paul, M. Epileptic seizure detection by analyzing EEG signals using different transformation techniques. Neurocomputing 2014, 145, 190-200. [CrossRef]

8. Mursalin, M.; Zhang, Y.; Chen, Y.; Chawla, N.V. Automated epileptic seizure detection using improved correlation-based feature selection with random forest classifier. Neurocomputing 2017, 241, 204-214. [CrossRef]

9. Kumar, Y.; Dewal, M.L.; Anand, R.S. Epileptic seizure detection using DWT based fuzzy approximate entropy and support vector machine. Neurocomputing 2014, 133, 271-279. [CrossRef]

10. Dhiman, R.; Saini, J.S.; Priyanka Genetic algorithms tuned expert model for detection of epileptic seizures from EEG signatures. Appl. Soft Comput. 2014, 19, 8-17. [CrossRef] 
11. Samiee, K.; Kovacs, P.; Gabbouj, M. Epileptic Seizure Classification of EEG time-series using Rational Discrete Short Time Fourier Transform. IEEE Trans. Biomed. Eng. 2015, 62, 541-552. [CrossRef] [PubMed]

12. Chen, G. Automatic EEG seizure detection using dual-tree complex wavelet-Fourier features. Expert Syst. Appl. 2014, 41, 2391-2394. [CrossRef]

13. Song, J.L.; Hu, W.; Zhang, R. Automated detection of epileptic EEGs using a novel fusion feature and extreme learning machine. Neurocomputing 2016, 175, 383-391. [CrossRef]

14. Chen, D.; Wan, S.; Bao, F.S. Epileptic Focus Localization Using Discrete Wavelet Transform Based on Interictal Intracranial EEG. IEEE Trans. Neural Syst. Rehabil. Eng. 2017, 25, 413-425. [CrossRef] [PubMed]

15. Kocadagli, O.; Langari, R. Classification of EEG signals for Epileptic Seizures using hybrid Artificial Neural Network based Wavelet Transforms and Fuzzy Relations. Expert Syst. Appl. 2017, 88, 419-434. [CrossRef]

16. Li, D.; Xie, Q.; Jin, Q.; Hirasawa, K. A sequential method using multiplicative extreme learning machine for epileptic seizure detection. Neurocomputing 2016, 214, 692-707. [CrossRef]

17. Han, J.; Li, Y.; Kang, J.; Cai, E.; Tong, Z.; Ouyang, G.; Li, X. Global Synchronization of Multichannel EEG Based on Rényi Entropy in Children with Autism Spectrum Disorder. Appl. Sci. 2017, 7, 257. [CrossRef]

18. Pedram, M.Z.; Shamloo, A.; Alasty, A.; Ghafar-Zadeh, E. Toward Epileptic Brain Region Detection Based on Magnetic Nanoparticle Patterning. Sensors 2015, 15, 24409-24427. [CrossRef] [PubMed]

19. Bhattacharyya, A.; Pachori, R.B.; Upadhyay, A.; Acharya, U.R. Tunable-Q Wavelet Transform Based Multiscale Entropy Measure for Automated Classification of Epileptic EEG Signals. Appl. Sci. 2017, 7, 385. [CrossRef]

20. Gottschalk, S.; Fehm, T.F.; Deán-Ben, X.L.; Tsytsarev, V.; Razansky, D. Correlation between volumetric oxygenation responses and electrophysiology identifies deep thalamocortical activity during epileptic seizures. Neurophotonics 2017, 4, 011007. [CrossRef] [PubMed]

21. Li, M.A.; Zhu, W.; Liu, H.N.; Yang, J.F. Adaptive Feature Extraction of Motor Imagery EEG with Optimal Wavelet Packets and SE-Isomap. Appl. Sci. 2017, 7, 390. [CrossRef]

22. Sieu, L.A.; Bergel, A.; Tiran, E.; Deffieux, T.; Pernot, M.; Gennisson, J.L.; Tanter, M.; Cohen, I. EEG and functional ultrasound imaging in mobile rats. Nat. Methods 2015, 12, 831-834. [CrossRef] [PubMed]

23. Mu, Z.; Hu, J.; Min, J. Driver Fatigue Detection System Using Electroencephalography Signals Based on Combined Entropy Features. Appl. Sci. 2017, 7, 150. [CrossRef]

24. Macé, E.; Montaldo, G.; Cohen, I.; Baulac, M.; Fink, M.; Tanter, M. Functional ultrasound imaging of the brain. Nat. Methods 2013, 60, 492-506. [CrossRef] [PubMed]

25. Jiang, Y.; Wu, D.; Deng, Z.; Qian, P.; Wang, J.; Wang, G.; Chung, F.; Choi, K.Z.; Wang, S. Seizure Classification form EEG Signals using Transfer Learning, Semi-Supervised Learning and TSK Fuzzy System. IEEE Trans. Neural Syst. Rehabil. Eng. 2017, 25, 2270-2284. [CrossRef] [PubMed]

26. Zhang, T.; Chen, W. LMD based features for the automatic seizure detection of EEG signals using SVM. IEEE Trans. Neural Syst. Rehabil. Eng. 2017, 25, 1100-1108. [CrossRef] [PubMed]

27. Bose, R.; Chatterjee, S.; Raychoudhury, N. Detection of Epileptic Seizure and Seizure-free EEG Signals Employing Generalized S-Transform. IET Sci. Meas. Technol. 2017, 11, 847-855.

28. Amorim, P.; Moraes, T.; Fazanaro, D.; Silva, J.; Pedrini, H. Electroencephalogram signal classification based on shearlet and contourlet transforms. Expert Syst. Appl. 2017, 67, 140-147. [CrossRef]

29. Riaz, F.; Hassan, A.; Rehman, S.; Niazi, I.K.; Dremstrup, K. EMD-Based Temporal and Spectral Features for the Classification of EEG Signals Using Supervised Learning. IEEE Trans. Neural Syst. Rehabil. Eng. 2016, 24, $28-35$. [CrossRef] [PubMed]

30. Vidyaratne, L.S.; Iftekharuddin, K.M. Real-Time Epileptic Seizure Detection Using EEG. IEEE Trans. Neural Syst. Rehabil. Eng. 2017, 25, 2146-2156. [CrossRef] [PubMed]

31. Li, Y. A novel statistical algorithm for multiclass EEG signal classification. Eng. Appl. Artif. Intell. 2014, 34, 154-167.

32. Fei, K.; Wang, W.; Yang, Q.; Tang, S. Chaos Feature Study in Fractional Fourier Domain for Preictal Prediction of Epileptic Seizure. Neurocomputing 2017, 249, 290-298. [CrossRef]

33. Alçin, M.F.; Siuly, S.; Bajaj, V.; Guo, Y.; Sengür, A.; Zhang, Y. Multi-category EEG signal classification developing time-frequency textures based Fisher Vector encoding method. Neurocomputing 2016, 218, 251-258. [CrossRef]

34. Li, Y.; Luo, M.L.; Li, K. A multiwavelet-based time-varying model identification approach for time-frequency analysis of EEG signals. Neurocomputing 2016, 193, 106-114. [CrossRef] 
35. Sharma, R.; Pachori, R.B. Classification of epileptic seizures in EEG signals based on phase space representation of intrinsic mode functions. Expert Syst. Appl. 2015, 42, 1106-1117. [CrossRef]

36. Martinez-Del-Rincon, J.; Santofimia, M.J.; Toro, X.D.; Barba, J.; Romero, F.; Navas, P.; Lopez, J.C. Non-linear classifiers applied to EEG analysis for epilepsy seizure detection. Expert Syst. Appl. 2017, 86, 99-112. [CrossRef]

37. Raghu, S.; Sriraam, N. Optimal configuration of multilayer perceptron neural network classifier for recognition of intracranial epileptic seizures. Expert Syst. Appl. 2017, 89, 205-221. [CrossRef]

38. Dhiman, R.; Saini, J.S. Bio-geography Based Hybrid Scheme for Automatic Detection of Epileptic Seizures form EEG Signatures. Appl. Soft Comput. 2017, 51, 116-129. [CrossRef]

39. Wang, G.; Deng, Z.; Choi, K.S. Detection of Epilepsy with Electroencephalogram using Rule-based Classifiers. Neurocomputing 2017, 228, 283-290. [CrossRef]

40. Diykh, M.; Li, Y.; Wen, P. Classify Epileptic EEG Signals Using Weighted Complex Networks Based Community Structure Detection. Expert Syst. Appl. 2017, 90, 87-100. [CrossRef]

41. Li, Y.; Wang, X.; Luo, L.; Li, K.; Yang, X.; Guo, Q. Epileptic Seizure Classification of EEGs using Time-Frequency Analysis based Multiscale Radial Basis Functions. IEEE J. Biomed. Health 2018, 22, 386-397. [CrossRef] [PubMed]

42. Wang, G.; Sun, Z.; Tao, R.; Li, K.; Bao, G.; Yan, X. Epileptic Seizure Detection Based on Partial Directed Coherence Analysis. IEEE J. Biomed. Health 2016, 20, 873-879. [CrossRef] [PubMed]

43. Li, Y.; Wei, H.L.; Billings, S.A.; Sarrigiannis, P.G. Time-varying model identification for time-frequency feature extraction from EEG data. J. Neurosci. Meth. 2011, 196, 151-158. [CrossRef] [PubMed]

44. Rosenow, F.; Klein, K.M.; Hamer, H.M. Non-invasive EEG evaluation in epilepsy diagnosis. Expert Rev. Neurother. 2015, 15, 425-444. [CrossRef] [PubMed]

45. Nagaraj, S.B.; Stevenson, N.J.; Marnane, W.P.; Boylan, G.B. Neonatal seizure detection using atomic decomposition with a novel dictionary. IEEE Trans. Biomed. Eng. 2014, 61, 2724-2732. [CrossRef] [PubMed]

46. Wu, H.; Wang, Y.; Huang, J. Identification of Electronic Disguised Voices. IEEE Trans. Inf. Forensics Secur. 2014, 9, 489-500. [CrossRef]

47. Montero, J.M.; Barra-Chicote, R. Feature extraction from smart-phone inertial signals for human activity segmentation. Singal Process. 2016, 120, 359-3725.

48. Ghaderyan, P.; Abbasi, A. A novel cepstral-based technique for automatic cognitive load estimation. Biomed. Signal Process. Control 2018, 39, 396-404. [CrossRef]

49. Hua, G.; Goh, J.; Thing, V.L.L. Cepstral Analysis for the Application of Echo-Based Audio Watermark Detection. IEEE Trans. Inf. Forensics Secur. 2015, 10, 1850-1861.

50. Li, Y.; Liu, Q.; Tan, S.R.; Chan, R.H.M. High-resolution time-frequency analysis of EEG signals using multiscale radial basis functions. Neurocomputing 2016, 195, 96-103. [CrossRef]

51. Jäntti, J.; Chaudhari, S.; Koivunen, V. Detection and Classification of OFDM Waveforms Using Cepstral Analysis. IEEE Trans. Signal Process. 2015, 63, 4284-4299. [CrossRef]

52. Colominas, M.A.; El, M.S.H.J.; Jrad, N.; Humeau-Heurtier, A.; Bogaert, P.V. Time-varying Time-frequency Complexity Measures for Epileptic EEG Data Analysis. IEEE Trans. Biomed. Eng. 2018, 65, 1681-1688. [PubMed]

53. Wang, N.; Lyu, M.R. Extracting and Selecting Distinctive EEG Features for Efficient Epileptic Seizure Prediction. IEEE J. Biomed. Health 2015, 19, 1648-1659. [CrossRef] [PubMed]

54. Ekong, U.; Lam, H.K.; Xiao, B.; Ouyang, G.; Liu, H.; Chan, K.Y.; Ling, S.H. Classification of epilepsy seizure phase using interval type-2 fuzzy support vector machines. Neurocomputing 2016, 199, 66-76. [CrossRef]

55. Daou, H.; Labeau, F. Dynamic Dictionary for Combined EEG Compression and Seizure Detection. IEEE J. Biomed. Health 2014, 18, 247-256. [CrossRef] [PubMed]

56. Bhattacgaryya, A.; Pachori, R.B. A multivariate approach for patient specific EEG seizure detection using empirical wavelet transform. IEEE Trans. Biomed. Eng. 2017, 64, 2003-2015. [CrossRef] [PubMed]

57. Islam, M.K.; Rastegarnia, A.; Yang, Z. A Wavelet-Based Artifact Reduction From Scalp EEG for Epileptic Seizure Detection. IEEE J. Biomed. Health 2016, 20, 1321-1332. [CrossRef] [PubMed]

58. Sharma, M.; Dhere, A.; Pachori, R.B.; Acharya, U.R. An automatic detection of focal EEG signals using new class of time-frequency localized orthogonal wavelet filter banks. Knowl. Based Syst. 2017, 118, $217-227$. [CrossRef] 
59. Kikove, M.; Francois, C.; Verly, J. Comparative evaluation of existing and new methods for correcting ocular artifacts in electroencephalographic recordings. Singal Process. 2014, 98, 102-120. [CrossRef]

60. Lam, H.K.; Ekong, U.; Xiao, B.; Ouyang, G.; Liu, H.; Chan, K.Y.; Ling, S.H. Variable weight neural networks and their applications on material surface and epilepsy seizure phase classifications. Neurocomputing 2015, 149, 1177-1187. [CrossRef]

(C) 2018 by the authors. Licensee MDPI, Basel, Switzerland. This article is an open access article distributed under the terms and conditions of the Creative Commons Attribution (CC BY) license (http://creativecommons.org/licenses/by/4.0/). 